Purdue University Purdue e-Pubs

2009

\title{
Rarefied Gas Flow in Microtubes at Different Inlet- Outlet Pressure Ratios
}

\author{
Z. Yang \\ Purdue University - Main Campus \\ S V. Garimella \\ Purdue Univers, sureshg@purdue.edu
}

Follow this and additional works at: http://docs.lib.purdue.edu/coolingpubs

Yang, Z. and Garimella, S V., "Rarefied Gas Flow in Microtubes at Different Inlet-Outlet Pressure Ratios" (2009). CTRC Research Publications. Paper 125.

http://docs.lib.purdue.edu/coolingpubs/125

This document has been made available through Purdue e-Pubs, a service of the Purdue University Libraries. Please contact epubs@purdue.edu for additional information. 


\title{
Rarefied gas flow in microtubes at different inlet-outlet pressure ratios
}

\author{
Z. Yang and S. V. Garimella $a^{a)}$ \\ Cooling Technologies Research Center, School of Mechanical Engineering and Birck Nanotechnology \\ Center, Purdue University, West Lafayette, Indiana 47907-2088, USA
}

(Received 22 October 2008; accepted 13 April 2009; published online 27 May 2009)

\begin{abstract}
A model is developed for rarefied gas flow in long microtubes with different inlet-outlet pressure ratios at low Mach numbers. The model accounts for significant changes in Knudsen number along the length of the tube and is therefore applicable to gas flow in long tubes encountering different flow regimes along the flow length. Predictions from the model show good agreement with experimental measurements of mass flow rate, pressure drop, and inferred streamwise pressure distribution obtained under different flow conditions and offer a better match with experiments than do those from a conventional slip flow model. (C) 2009 American Institute of Physics.
\end{abstract}

[DOI: 10.1063/1.3139310]

\section{INTRODUCTION}

Regimes of gas flow change with the Knudsen number $\mathrm{Kn}$, defined as the ratio of the mean free path to a geometric characteristic length such as channel width or tube radius. As the Knudsen number increases, gas flows sequentially experience four different regimes: continuum flow $(\mathrm{Kn}<0.001)$, slip flow $(0.001<\mathrm{Kn}<0.1)$, transition flow $(0.1<\mathrm{Kn}<10)$, and free molecular flow $(\mathrm{Kn}>10)$. Detailed information on flow classification can be found in Ref. 1.

Rarefied gas flows generally refer to flows with Kn exceeding 0.001, and their flow behavior is not well predicted by the continuum-based Navier-Stokes equations. Since Knudsen number only reflects the relative relationship between the molecular mean free path and a characteristic length of the geometry in question, a specific Kn may represent different flow scenarios. For instance, a gas flow characterized by $\mathrm{Kn}=1$ may either signify a low pressure condition (and thus a large mean free path) in macroscale channels or a higher pressure flow in very small channels. Research into rarefied gas flows has thus considered two major directions: low-density flows in near-vacuum environments and dense flows in micro-/nanostructures.

Early interest in studying rarefied gas flows originated from vacuum science research, with applications in vacuum and orbital technologies. A good discussion of this area is available in Ref. 2. With the recent rapid progress in micro-/ nanotechnologies, rarefied gaseous flows in small structures such as microdevices and microchannels has drawn increasing attention. ${ }^{3-6}$

The one-dimensional model of Prud'homme et al. ${ }^{7}$ of gas flow in a straight tube showed that gas compressibility caused a nonconstant pressure gradient along the flow direction; rarefaction effects were not considered in this analysis. Investigations of gas flow in two-dimensional (2D) microchannels using the first term in the series expansion of slip velocity in terms of Knudsen number (which accounts for rarefaction effects) revealed the contribution of the slip velocity on mass flow and pressure drop. ${ }^{3,4}$ The numerical so-

\footnotetext{
${ }^{a)}$ Author to whom correspondence should be addressed. Telephone: (765) 494-5621. Electronic mail: sureshg@ @urdue.edu.
}

lution in Ref. 5 for slightly rarefied gas flow, i.e., $\mathrm{Kn}<0.2$, indicated that mass flow rate may be increased both due to slip and thermal creep effects. For highly rarefied flows, in which the deviation from Navier-Stokes predictions is significant, the direct simulation Monte Carlo (DSMC) method has been used. ${ }^{8-12}$ DSMC is a particle-based method and is especially efficient in numerical solutions of transition flow. A hybrid DSMC and Navier-Stokes scheme ${ }^{13}$ as well as hybrid continuum-particle methods based on computational fluid dynamics and DSMC (Ref. 14) have also been developed. The Boltzmann transport equation has also been used to study highly rarefied flows. ${ }^{15,16}$ The Boltzmann equation describes the statistical distribution of a particle in a fluid and is one of the most important equations of nonequilibrium statistical mechanics. To account for the collision integral, the Bhatnagar-Gross-Krook model ${ }^{17}$ as well as the hardsphere and Maxwellian models ${ }^{18,19}$ have been used extensively. Solutions of the Boltzmann equation have been obtained for a cylindrical geometry, ${ }^{20}$ as well as for ducts of different cross sections. ${ }^{18,21}$

Experimental studies of rarefied gas flows have advanced rapidly due to developments in micromachining and lithographic techniques. Microchannels as small as $0.5 \mu \mathrm{m}$ in depth can be fabricated in silicon substrates such that a relatively high Knudsen number can be achieved under normal experimental conditions. ${ }^{22}$ While overall pressure drop and flow rate have been relatively easy to measure, measurement of the streamwise pressure distribution along the flow direction has been more challenging. With the recent development of techniques to fabricate pressure sensors directly into microchannels on a chip, experimental results have been obtained which show a nonlinear pressure drop along the channel due to gas compressibility effects. ${ }^{6,22}$

Although significant progress has been made in the study of rarefied gas flows, models which consider gas flows in which the Knudsen number changes significantly along the flow direction are lacking. Under such conditions, several flow regimes may coexist along the length of the channel. Past studies mainly focused on channel flows with relatively small pressure ratios between the inlet and outlet so that the Knudsen number did not change significantly along the 
channel. However, rarefied flows with large pressure ratios are frequently encountered in practice in applications such as a gas flowing from atmospheric conditions into a vacuum chamber wherein regimes ranging from continuum to free molecular flow may be experienced. Similarly, gas flows in microchannels may undergo a large pressure drop to overcome the increased flow resistance at small scales. The Knudsen number in such cases can vary greatly from the inlet to outlet due to the large variations in pressure. The present work presents a study of rarefied gas flows over a range of pressure ratios between the inlet and outlet. A model is developed to predict mass flow rate and streamwise pressure distribution for different pressure ratios and Knudsen numbers. The model is validated against experimental results in the transitional flow regime obtained as part of this work, as well as solutions for the linearized Boltzmann equation (at a low pressure ratio) in a cylindrical tube over the entire flow regime. ${ }^{20}$ The good agreement obtained in these comparisons shows that the model is valid for predicting mass flow rate and streamwise pressure distribution for gas flows up to the transitional regime and is potentially applicable over the full range of flow regimes.

\section{MODEL DEVELOPMENT}

\section{A. Governing equations}

We consider a steady, constant-temperature, compressible, and axisymmetric gas flow in a long capillary tube, with the effect of gravity being negligible. The 2D governing equations in cylindrical coordinates, with the Burnett equations ${ }^{23}$ used for the stress tensor in the momentum equations, are written as follows:

(a) continuity equation,

$$
\frac{\partial(\rho v)}{\partial r}+\frac{\rho v}{r}+\frac{\partial(\rho u)}{\partial x}=0,
$$

(b) $x$-momentum equation in the axial direction,

$$
\begin{aligned}
\rho\left(v \frac{\partial u}{\partial r}\right. & \left.+u \frac{\partial u}{\partial x}\right) \\
= & -\frac{\partial p}{\partial x}+\mu\left(\frac{4}{3} \frac{\partial^{2} u}{\partial x^{2}}+\frac{\partial^{2} u}{\partial r^{2}}+\frac{1}{3} \frac{\partial^{2} v}{\partial x \partial r}+\frac{1}{3 r} \frac{\partial v}{\partial x}+\frac{1}{r} \frac{\partial u}{\partial r}\right) \\
& -\left(\frac{\partial \sigma_{r x}}{\partial r}+\frac{\sigma_{r x}}{r}+\frac{\partial \sigma_{x x}}{\partial x}\right),
\end{aligned}
$$

and

(c) $r$-momentum equation in the radial direction,

$$
\begin{aligned}
\rho\left(v \frac{\partial v}{\partial r}\right. & \left.+u \frac{\partial v}{\partial x}\right) \\
= & -\frac{\partial p}{\partial r}+\mu\left(\frac{4}{3} \frac{\partial^{2} v}{\partial r^{2}}+\frac{\partial^{2} v}{\partial x^{2}}+\frac{1}{3} \frac{\partial^{2} u}{\partial x \partial r}+\frac{4}{3 r} \frac{\partial v}{\partial r}-\frac{4}{3} \frac{v}{r^{2}}\right) \\
& -\left(\frac{\partial \sigma_{r r}}{\partial r}+\frac{\sigma_{r r}-\sigma_{\theta \vartheta}}{r}+\frac{\partial \sigma_{r x}}{\partial x}\right),
\end{aligned}
$$

where $x$ and $r$ are the coordinates in the axial and radial directions, respectively, $u$ and $v$ are the velocities in the $x$ and $r$ directions, respectively, $p$ is the pressure, $\rho$ and $\mu$ are the density and viscosity of the gas, respectively, and $\sigma_{i, j}$ are components of the second-order constitutive tensor in the Burnett equation derived by the Chapman-Enskog method. ${ }^{24}$ Expressions for $\sigma_{i, j}$ are provided in the Appendix. The fluid considered here is assumed to be an ideal gas.

We further define the following normalized variables:

$$
\begin{aligned}
& \bar{x}=x / L, \quad \bar{r}=r / r_{0}, \quad \bar{u}=u / u_{o}, \quad \bar{v}=v / u_{o}, \\
& \bar{p}=p / p_{o}, \quad \bar{\rho}=\rho / \rho_{o},
\end{aligned}
$$

where $L$ is the tube length, $r_{0}$ the tube radius, $u_{o}$ the mean axial velocity at the outlet, and $p_{o}$ and $\rho_{o}$ the pressure and gas density at the outlet, respectively. Introducing these dimensionless variables into Eqs. (1)-(3) yields the following nondimensional governing equations:

(a) continuity equation,

$$
\frac{\partial(\bar{p} \bar{v})}{\partial \bar{r}}+\frac{\bar{p} \bar{v}}{\bar{r}}+\varepsilon \frac{\partial(\bar{p} \bar{u})}{\partial \bar{x}}=0,
$$

(b) axial momentum equation,

$$
\begin{aligned}
& \frac{16 \gamma^{1 / 2}}{5 \sqrt{2 \pi}} \frac{\mathrm{Ma}_{o}}{\mathrm{Kn}_{o}} \bar{p}\left(\bar{v} \frac{\partial \bar{u}}{\partial \bar{r}}+\varepsilon \cdot \bar{u} \frac{\partial \bar{u}}{\partial \bar{x}}\right)=-\frac{16}{5 \sqrt{2 \pi \gamma}} \frac{\varepsilon}{\mathrm{Kn}_{o} \mathrm{Ma}_{o}} \frac{\partial \bar{p}}{\partial \bar{x}}+\varepsilon^{2} \frac{4}{3} \frac{\partial^{2} \bar{u}}{\partial \bar{x}^{2}}+\frac{\partial^{2} \bar{u}}{\partial \bar{r}^{2}}+\varepsilon \frac{1}{3} \frac{\partial^{2} \bar{v}}{\partial \bar{x} \partial \bar{r}}+\varepsilon \frac{1}{3 \bar{r}} \frac{\partial \bar{v}}{\partial \bar{x}}+\frac{1}{\bar{r}} \frac{\partial \bar{u}}{\partial \bar{r}} \\
& +\varepsilon \frac{5 \sqrt{2 \pi \gamma}}{16} \mathrm{Kn}_{o} \mathrm{Ma}_{o}\left\{\frac{1}{\bar{p}^{2}} \frac{\partial \bar{p}}{\partial \bar{x}}\left[\left(\frac{\omega_{2}}{3}+\frac{\omega_{6}}{12}\right)\left(\frac{\partial \bar{u}}{\partial \bar{r}}\right)^{2}+O(\varepsilon)\right]-\frac{1}{\bar{p}}\left[2\left(\frac{\omega_{2}}{3}+\frac{\omega_{6}}{12}\right) \frac{\partial \bar{u}}{\partial \bar{r}} \frac{\partial^{2} \bar{u}}{\partial \bar{r}} \partial \bar{x}\right.\right. \\
& +O(\varepsilon)]\}+\varepsilon \cdot \omega_{2} \frac{5 \sqrt{2 \pi}}{16 \sqrt{\gamma}} \frac{\mathrm{Kn}_{o}}{\mathrm{Ma}_{o}}\left\{-\frac{1}{\bar{p}^{2}} \frac{\partial \bar{p}}{\partial \bar{x}}\left[\varepsilon^{2} \frac{2}{3} \frac{\partial}{\partial \bar{x}}\left(\frac{1}{\bar{p}} \frac{\partial \bar{p}}{\partial \bar{x}}\right)-\frac{1}{3 \bar{r} \bar{p}} \frac{\partial \bar{p}}{\partial \bar{r}}-\frac{1}{3} \frac{\partial}{\partial \bar{r}}\left(\frac{1}{\bar{p}} \frac{\partial \bar{p}}{\partial \bar{r}}\right)\right]\right. \\
& \left.+\frac{1}{\bar{p}} \frac{\partial}{\partial \bar{x}}\left[\varepsilon^{2} \frac{2}{3} \frac{\partial}{\partial \bar{x}}\left(\frac{1}{\bar{p}} \frac{\partial \bar{p}}{\partial \bar{x}}\right)-\frac{1}{3 \bar{r} \bar{p}} \frac{\partial \bar{p}}{\partial \bar{r}}-\frac{1}{3} \frac{\partial}{\partial \bar{r}}\left(\frac{1}{\bar{p}} \frac{\partial \bar{p}}{\partial \bar{r}}\right)\right]\right\} \\
& +\frac{5 \sqrt{2 \pi \gamma}}{16} \mathrm{Kn}_{o} \mathrm{Ma}_{o}\left\{\frac { 1 } { \overline { p } ^ { 2 } } \frac { \partial \overline { p } } { \partial \overline { r } } \left[\frac{\left(3 \omega_{1}-4 \omega_{2}+\omega_{6}\right)}{6} \frac{\partial \bar{u}}{\partial \bar{r}} \frac{\partial \bar{v}}{\partial \bar{r}}+\varepsilon \frac{\left(3 \omega_{1}-10 \omega_{2}+\omega_{6}\right)}{6} \frac{\partial \bar{u}}{\partial \bar{r}} \frac{\partial \bar{u}}{\partial \bar{x}}\right.\right.
\end{aligned}
$$




$$
\begin{aligned}
& \left.+\frac{\left(3 \omega_{1}-2 \omega_{2}-2 \omega_{6}\right)}{6} \frac{\partial \bar{u}}{\partial \bar{r}} \frac{\bar{v}}{\bar{r}}+O\left(\varepsilon^{2}\right)\right]-\frac{1}{\bar{p}} \frac{\partial}{\partial \bar{r}}\left[\frac{\left(3 \omega_{1}-4 \omega_{2}+\omega_{6}\right)}{6} \frac{\partial \bar{u}}{\partial \bar{r}} \frac{\partial \bar{v}}{\partial \bar{r}}\right. \\
& \left.\left.+\varepsilon \frac{\left(3 \omega_{1}-10 \omega_{2}+\omega_{6}\right)}{6} \frac{\partial \bar{u}}{\partial \bar{r}} \frac{\partial \bar{u}}{\partial \bar{x}}+\frac{\left(3 \omega_{1}-2 \omega_{2}-2 \omega_{6}\right)}{6} \frac{\partial \bar{u} \bar{v}}{\partial \bar{r}} \overline{\bar{r}}+O\left(\varepsilon^{2}\right)\right]\right\} \\
& +\varepsilon \cdot \omega_{2} \frac{5 \sqrt{2 \pi}}{16 \sqrt{\gamma}} \frac{\mathrm{Kn}_{o}}{\mathrm{Ma}_{o}}\left\{-\frac{1}{\bar{p}^{2}} \frac{\partial \bar{p}}{\partial \bar{r}}\left[\frac{\partial}{\partial \bar{r}}\left(\frac{1}{\bar{p}} \frac{\partial \bar{p}}{\partial \bar{x}}\right)+\frac{1}{3} \frac{\partial}{\partial \bar{x}}\left(\frac{1}{\bar{p}} \frac{\partial \bar{p}}{\partial \bar{r}}\right)\right]+\frac{1}{\bar{p}} \frac{\partial}{\partial \bar{r}}\left[\frac{\partial}{\partial \bar{r}}\left(\frac{1}{\bar{p}} \frac{\partial \bar{p}}{\partial \bar{x}}\right)\right.\right. \\
& \left.\left.+\frac{1}{3} \frac{\partial}{\partial \bar{x}}\left(\frac{1}{\bar{p}} \frac{\partial \bar{p}}{\partial \bar{r}}\right)\right]\right\}+\frac{5 \sqrt{2 \pi \gamma}}{16} \mathrm{Kn}_{o} \mathrm{Ma}_{o}\left\{-\frac{1}{\bar{p} \cdot \bar{r}}\left[\frac{\left(3 \omega_{1}-4 \omega_{2}+\omega_{6}\right)}{6} \frac{\partial \bar{u}}{\partial \bar{r}} \frac{\partial \bar{v}}{\partial \bar{r}}\right.\right. \\
& \left.\left.+\varepsilon \frac{\left(3 \omega_{1}-10 \omega_{2}+\omega_{6}\right)}{6} \frac{\partial \bar{u}}{\partial \bar{r}} \frac{\partial \bar{u}}{\partial \bar{x}}+\frac{\left(3 \omega_{1}-2 \omega_{2}-2 \omega_{6}\right)}{6} \frac{\partial \bar{u} \bar{v}}{\partial \bar{r}}+O\left(\varepsilon^{2}\right)\right]\right\}
\end{aligned}
$$

and

(c) radial momentum equation,

$$
\begin{aligned}
& \frac{16 \sqrt{\gamma}}{5 \sqrt{2 \pi}} \frac{\mathrm{Ma}_{o}}{\mathrm{Kn}_{o}} \bar{p}\left(\bar{v} \frac{\partial \bar{v}}{\partial \bar{r}}+\varepsilon \cdot \bar{u} \frac{\partial \bar{v}}{\partial \bar{x}}\right)=-\frac{16}{5 \sqrt{2 \pi \gamma}} \frac{1}{\mathrm{Kn}_{o} \mathrm{Ma}_{o}} \frac{\partial \bar{p}}{\partial \bar{r}}+\frac{4}{3} \frac{\partial^{2} \bar{v}}{\partial \bar{r}^{2}}+\varepsilon^{2} \frac{\partial^{2} \bar{v}}{\partial \bar{x}^{2}}+\varepsilon \frac{1}{3} \frac{\partial^{2} u}{\partial \bar{r} \partial \bar{x}}+\frac{4}{3 \bar{r}} \frac{\partial \bar{v}}{\partial \bar{r}}-\frac{4 \bar{v}}{3 \bar{r}^{2}} \\
& +\frac{5 \sqrt{2 \pi \gamma}}{16} \mathrm{Kn}_{o} \operatorname{Ma}_{o}\left\{\frac{1}{\bar{p}^{2}} \frac{\partial \bar{p}}{\partial \bar{r}}\left[\left(-\frac{2 \omega_{2}}{3}+\frac{\omega_{6}}{12}\right)\left(\frac{\partial \bar{u}}{\partial \bar{r}}\right)^{2}+O(\varepsilon)\right]-\frac{1}{\bar{p}}\left[2\left(-\frac{2 \omega_{2}}{3}+\frac{\omega_{6}}{12}\right) \frac{\partial \bar{u}}{\partial \bar{r}} \frac{\partial^{2} \bar{u}}{\partial \bar{r}^{2}}\right.\right. \\
& +O(\varepsilon)]\}+\omega_{2} \frac{5 \sqrt{2 \pi}}{16 \sqrt{\gamma}} \frac{\mathrm{Kn}_{o}}{\mathrm{Ma}_{o}}\left\{-\frac{1}{\bar{p}^{2}} \frac{\partial \bar{p}}{\partial \bar{r}}\left[\frac{2}{3} \frac{\partial}{\partial \bar{r}}\left(\frac{1}{\bar{p}} \frac{\partial \bar{p}}{\partial \bar{r}}\right)-\frac{1}{3 \bar{r} \bar{p}} \frac{\partial \bar{p}}{\partial \bar{r}}-\varepsilon^{2} \frac{1}{3} \frac{\partial}{\partial \bar{x}}\left(\frac{1}{\bar{p}} \frac{\partial \bar{p}}{\partial \bar{x}}\right)\right]\right. \\
& \left.+\frac{1}{\bar{p}} \frac{\partial}{\partial \bar{r}}\left[\frac{2}{3} \frac{\partial}{\partial \bar{r}}\left(\frac{1}{\bar{p}} \frac{\partial \bar{p}}{\partial \bar{r}}\right)-\frac{1}{3 \bar{r} \bar{p}} \frac{\partial \bar{p}}{\partial \bar{r}}-\varepsilon^{2} \frac{1}{3} \frac{\partial}{\partial \bar{x}}\left(\frac{1}{\bar{p}} \frac{\partial \bar{p}}{\partial \bar{x}}\right)\right]\right\} \\
& +\frac{5 \sqrt{2 \pi \gamma}}{16} \mathrm{Kn}_{o} \mathrm{Ma}_{o}\left\{\varepsilon \frac { 1 } { \overline { p } ^ { 2 } } \frac { \partial \overline { p } } { \partial \overline { x } } \left[\frac{\left(3 \omega_{1}-4 \omega_{2}+\omega_{6}\right)}{6} \frac{\partial \bar{u}}{\partial \bar{r}} \frac{\partial \bar{v}}{\partial \bar{r}}+\varepsilon \frac{\left(3 \omega_{1}-10 \omega_{2}+\omega_{6}\right)}{6} \frac{\partial \bar{u}}{\partial \bar{r}} \frac{\partial \bar{u}}{\partial \bar{x}}\right.\right. \\
& \left.+\frac{\left(3 \omega_{1}-2 \omega_{2}-2 \omega_{6}\right)}{6} \frac{\partial \bar{u}}{\partial \bar{r}} \frac{\bar{v}}{\bar{r}}+O\left(\varepsilon^{2}\right)\right]-\varepsilon \frac{1}{\bar{p}} \frac{\partial}{\partial \bar{x}}\left[\frac{\left(3 \omega_{1}-4 \omega_{2}+\omega_{6}\right)}{6} \frac{\partial \bar{u}}{\partial \bar{r}} \frac{\partial \bar{v}}{\partial \bar{r}}\right. \\
& \left.\left.+\varepsilon \frac{\left(3 \omega_{1}-10 \omega_{2}+\omega_{6}\right)}{6} \frac{\partial \bar{u}}{\partial \bar{r}} \frac{\partial \bar{u}}{\partial \bar{x}}+\frac{\left(3 \omega_{1}-2 \omega_{2}-2 \omega_{6}\right)}{6} \frac{\partial \bar{u}}{\partial \bar{r}} \frac{\bar{v}}{\bar{r}}+O\left(\varepsilon^{2}\right)\right]\right\} \\
& +\varepsilon^{2} \omega_{2} \frac{5 \sqrt{2 \pi}}{16 \sqrt{\gamma}} \frac{\mathrm{Kn}_{o}}{\mathrm{Ma}_{o}}\left\{-\frac{1}{\bar{p}^{2}} \frac{\partial \bar{p}}{\partial \bar{x}}\left[\frac{\partial}{\partial \bar{r}}\left(\frac{1}{\bar{p}} \frac{\partial \bar{p}}{\partial \bar{x}}\right)+\frac{1}{3} \frac{\partial}{\partial \bar{x}}\left(\frac{1}{\bar{p}} \frac{\partial \bar{p}}{\partial \bar{r}}\right)\right]+\frac{1}{\bar{p}} \frac{\partial}{\partial \bar{x}}\left[\frac{\partial}{\partial \bar{r}}\left(\frac{1}{\bar{p}} \frac{\partial \bar{p}}{\partial \bar{x}}\right)\right.\right. \\
& \left.\left.+\frac{1}{3} \frac{\partial}{\partial \bar{x}}\left(\frac{1}{\bar{p}} \frac{\partial \bar{p}}{\partial \bar{r}}\right)\right]\right\}+\frac{5 \sqrt{2 \pi \gamma}}{16} \mathrm{Kn}_{o} \mathrm{Ma}_{o}\left\{-\frac{1}{\bar{r} \bar{p}}\left[\left(-\omega_{2}+\frac{\omega_{6}}{4}\right)\left(\frac{\partial \bar{u}}{\partial \bar{r}}\right)^{2}+O(\varepsilon)\right]\right\} \\
& +\omega_{2} \frac{5 \sqrt{2 \pi}}{16 \sqrt{\gamma}} \frac{\mathrm{Kn}_{o}}{\mathrm{Ma}_{o}}\left\{\frac{1}{\bar{r} \bar{p}}\left[\frac{\partial}{\partial \bar{r}}\left(\frac{1}{\bar{p}} \frac{\partial \bar{p}}{\partial \bar{r}}\right)-\frac{1}{\bar{r} \bar{p}} \frac{\partial \bar{p}}{\partial \bar{r}}\right]\right\} \text {, }
\end{aligned}
$$

where $\varepsilon=r_{0} / L$, i.e., the ratio of the tube radius to its length, and $\gamma$ is the ratio of gas specific heats. The coefficients $\omega_{1}$, $\omega_{2}$, and $\omega_{6}$ depend on the gas model. For the hard-sphere gas model, $\omega_{1}=4.056, \omega_{2}=2.028$, and $\omega_{6}=7.424 .^{23}$ Mach number and Knudsen number are defined in terms of the gas properties at the outlet, i.e.,

$$
\mathrm{Ma}_{o}=\frac{u_{o}}{c}, \quad \mathrm{Kn}_{o}=\frac{\lambda_{o}}{r_{0}},
$$

where $c$ is the acoustic velocity and $\lambda_{o}$ the mean free path at the outlet. These two parameters can, respectively, be expressed as 


$$
c=\sqrt{\gamma R T} \quad \text { and } \quad \lambda_{o}=\frac{16 \mu_{0}}{5 \rho_{o} \sqrt{2 \pi R T}},
$$

where $R=287 \mathrm{~J} \mathrm{~kg}^{-1} \mathrm{~K}^{-1}$ is the gas constant of air, $T$ the temperature, and $\mu_{0}$ the viscosity of bulk gas.

\section{B. Scale analysis}

Since we consider gas flows in very long tubes, the value of $\varepsilon$ can be very small. In the experiments of this work, $\varepsilon$ ranges from $3.03 \times 10^{-4}$ to $4.10 \times 10^{-3}$. From Eq. (4), it is clear that $\bar{u}$ is of $O(1)$ while $\bar{v}$ is of $O(\varepsilon)$. In Eq. (5), the largest terms with $O(1)$ are the velocity derivatives $\partial^{2} \bar{u} / \partial \bar{r}^{2}$ and $(\partial \bar{u} / \partial \bar{r}) / \bar{r}$, so that the order of $\mathrm{Ma}_{o}$ is $O(\varepsilon)$ in order to ensure that the pressure gradient $\partial \bar{p} / \partial \bar{x}$ in the first term on the right hand side is of $O(1)$. Mach number being of $O(\varepsilon)$ is consistent with the fact that increasing the tube length at a fixed pressure drop will decrease the flow rate as well as Mach number. Knudsen number is typically considered to be of $O(1)$ for rarefied flows near the transition flow regime. Neglecting higher-order terms in $\varepsilon$, Eq. (6) can be reduced to

$$
\begin{aligned}
O(\varepsilon)= & -\frac{16}{5 \sqrt{2 \pi \gamma}} \frac{1}{\mathrm{Kn}_{o} \mathrm{Ma}_{o}} \frac{\partial \bar{p}}{\partial \bar{r}}+\frac{5 \sqrt{2 \pi \gamma}}{16} \mathrm{Kn}_{o} \mathrm{Ma}_{o}\left\{\frac{1}{\bar{p}^{2}} \frac{\partial \bar{p}}{\partial \bar{r}}\left[\left(-\frac{2 \omega_{2}}{3}+\frac{\omega_{6}}{12}\right)\left(\frac{\partial \bar{u}}{\partial \bar{r}}\right)^{2}\right]-\frac{1}{\bar{p}}\left[2\left(-\frac{2 \omega_{2}}{3}+\frac{\omega_{6}}{12}\right) \frac{\partial \bar{u}}{\partial \bar{r}} \frac{\partial^{2} \bar{u}}{\partial \bar{r}^{2}}\right]\right\} \\
& +\omega_{2} \frac{5 \sqrt{2 \pi}}{16 \sqrt{\gamma}} \frac{\mathrm{Kn}_{o}}{\mathrm{Ma}_{o}}\left\{-\frac{1}{\bar{p}} \frac{\partial \bar{p}}{\partial \bar{r}}\left[\frac{2}{3} \frac{\partial}{\partial \bar{r}}\left(\frac{1}{\bar{p}} \frac{\partial \bar{p}}{\partial \bar{r}}\right)-\frac{1}{3 \bar{r} \bar{p}} \frac{\partial \bar{p}}{\partial \bar{r}}\right]+\frac{1}{\bar{p}} \frac{\partial}{\partial \bar{r}}\left[\frac{2}{3} \frac{\partial}{\partial \bar{r}}\left(\frac{1}{\bar{p}} \frac{\partial \bar{p}}{\partial \bar{r}}\right)-\frac{1}{3 \bar{r} \bar{p}} \frac{\partial \bar{p}}{\partial \bar{r}}\right]\right\} \\
& +\frac{5 \sqrt{2 \pi \gamma}}{16} \mathrm{Kn}_{o} \mathrm{Ma}_{o}\left\{-\frac{1}{\bar{r} \bar{p}}\left[\left(-\omega_{2}+\frac{\omega_{6}}{4}\right)\left(\frac{\partial \bar{u}}{\partial \bar{r}}\right)^{2}\right]\right\}+\omega_{2} \frac{5 \sqrt{2 \pi}}{16 \sqrt{\gamma}} \frac{\mathrm{Kn}_{o}}{\mathrm{Ma}_{o}}\left\{\frac{1}{\bar{r} \bar{p}}\left[\frac{\partial}{\partial \bar{r}}\left(\frac{1}{\bar{p}} \frac{\partial \bar{p}}{\partial \bar{r}}\right)-\frac{1}{\bar{r} \bar{p}} \frac{\partial \bar{p}}{\partial \bar{r}}\right]\right\} .
\end{aligned}
$$

Since $\mathrm{Ma}_{o}$ is of $O(\varepsilon)$, the coefficient of the pressure derivative $\partial \bar{p} / \partial \bar{r}$ in the first term on the first right hand side of Eq. (7) is of $O(1 / \varepsilon)$, which implies that $\partial \bar{p} / \partial \bar{r}$ must be of order $O\left(\varepsilon^{2}\right)$. Retaining only those terms of order $O(1)$ in $\bar{u}, \bar{v}$, $\partial \bar{p} / \partial \bar{x}, \partial \bar{p} / \partial \bar{r}, \mathrm{Ma}_{o}$ and $\mathrm{Kn}_{o}$, Eq. (5) can be greatly simplified to

$$
0=-\frac{16}{5 \sqrt{2 \pi \gamma}} \frac{\varepsilon}{\mathrm{Kn}_{o} \mathrm{Ma}_{o}} \frac{\partial \bar{p}}{\partial \bar{x}}+\frac{\partial^{2} \bar{u}}{\partial \bar{r}^{2}}+\frac{1}{\bar{r}} \frac{\partial \bar{u}}{\partial \bar{r}}+O\left(\varepsilon^{2}\right) .
$$

\section{Expressions for mass flow rate and streamwise pressure distribution}

The boundary conditions on Eq. (8) are as follows:

(a) an axisymmetric boundary condition at the center,

$$
\left.\frac{\partial \bar{u}}{\partial \bar{r}}\right|_{\bar{r}=0}=0,
$$

and wall,

(b) a second-order slip boundary condition at the tube

$$
\left.\bar{u}\right|_{\bar{r}=1}=-\left.\frac{2-\sigma}{\sigma} \frac{\mathrm{Kn}}{1-b \mathrm{Kn}} \frac{\partial \bar{u}}{\partial \bar{r}}\right|_{\bar{r}=1},
$$

where $b=-1$ for fully developed flow, ${ }^{25}$ and the accommodation coefficient $\sigma$ is assumed to be 1 (a diffuse reflection boundary) for air flow over the glass surface inside capillary tubes. Here, $\mathrm{Kn}$ is the local Knudsen number and is distinct from $\mathrm{Kn}_{o}$ at the outlet. Ignoring higher-order terms and integrating Eq. (8) twice from $\bar{r}=0$ to 1 yields

$$
\bar{u}=\frac{8}{5 \sqrt{2 \pi \gamma}} \frac{\varepsilon}{\mathrm{Kn}_{o} \mathrm{Ma}_{o}} \frac{\partial \bar{p}}{\partial \bar{x}}\left(\frac{\bar{r}^{2}}{2}-\frac{\mathrm{Kn}}{1-b \mathrm{Kn}}-\frac{1}{2}\right) .
$$

Equation (11) implies that gas flows in long tubes have a parabolic velocity profile with a velocity jump at the wall, and such a profile has been validated for tube flow in all the flow regimes. ${ }^{25}$ Integrating Eq. (11) over the cross section yields the mass flow rate

$$
\begin{aligned}
\bar{Q} & =\frac{Q}{\rho_{o} u_{o} r_{0}^{2}} \\
& =\int_{\bar{r}=0}^{\bar{r}=1} 2 \pi \bar{r} \bar{\rho} \bar{u} \cdot d \bar{r} \\
& =-\frac{4 \sqrt{2 \pi}}{5 \sqrt{\gamma}} \frac{\varepsilon \cdot \bar{p}}{\mathrm{Kn}_{o} \mathrm{Ma}_{o}} \frac{d \bar{p}}{d \bar{x}}\left(\frac{1}{4}+\frac{\mathrm{Kn}}{1-b \mathrm{Kn}}\right) .
\end{aligned}
$$

Rewriting Eq. (12) in its dimensional form, we get

$$
Q=-\frac{\pi r_{0}^{4} p}{2 \mu R T} \frac{d p}{d x}\left(\frac{1}{4}+\frac{\mathrm{Kn}}{1-b \mathrm{Kn}}\right) .
$$

The viscosity $\mu$ changes with $\mathrm{Kn}$ as ${ }^{25}$

$$
\mu=\frac{\mu_{0}}{1+\alpha \mathrm{Kn}},
$$


where $\mu_{0}$ is the viscosity at the macroscale and $\alpha$ changes with $\mathrm{Kn}$ as

$$
\alpha=\frac{128}{3 \pi^{2}(1-4 / b)} \tan ^{-1}\left(4.0 \mathrm{Kn}^{0.4}\right) .
$$

In Eq. (14), it is apparent that $\mu$ approaches $\mu_{0}$ when the Knudsen number is very small. Substituting Eq. (14) into Eq. (13) yields

$$
\begin{aligned}
Q & =-\frac{\pi r_{0}^{4} p}{2 \mu_{0} R T} \frac{d p}{d x}\left(\frac{1}{4}+\frac{\mathrm{Kn}}{1-b \mathrm{Kn}}\right)(1+\alpha \mathrm{Kn}) \\
& =-\frac{\pi r_{0}^{4} p}{2 \mu_{0} R T} \frac{d p}{d x}\left(\frac{1}{4}+\frac{\mathrm{Kn}_{o} p_{o}}{p-b \mathrm{Kn}_{o} p_{o}}\right)\left(1+\frac{\alpha \mathrm{Kn}_{o} p_{o}}{p}\right) .
\end{aligned}
$$

Integrating Eq. (16) from the inlet $(x=0)$ to the outlet $(x=L)$ of the channel yields

$$
\begin{aligned}
Q= & \frac{\pi r_{0}^{4} p_{o}^{2}}{2 \mu_{0} R T L}\left[\frac{P^{2}-1}{8}+\frac{\mathrm{Kn}_{o}(4+\alpha)}{4}(P-1)\right. \\
& \left.+(b+\alpha) \mathrm{Kn}_{o}^{2} \ln \left(\frac{P-b \mathrm{Kn}_{o}}{1-b \mathrm{Kn}_{o}}\right)\right],
\end{aligned}
$$

where $P=p_{i} / p_{o}$ is the pressure ratio between the inlet and outlet. It is noted that in the derivation of Eq. (17), $\alpha$ is treated as a constant, since an explicit expression would not result if the functional dependence of $\alpha$ from Eq. (15) is used in the integration of Eq. (16) Thus, Eq. (17) is recommended for prediction of mass flow rate with near-constant Knudsen number along the tube, or in other words, with a small pressure ratio between the inlet and outlet.

For rarefied flows in long tubes, the pressure changes significantly in the flow direction as does the Knudsen number, which causes the value of $\alpha$ to change along the tube. We now develop a model that is applicable for rarefied gas flow with significant pressure variation, or large pressure ratios between the inlet and outlet. We retain the form of Eq. (17) and seek an appropriate function in place of the functional dependence of $\alpha$ in Eq. (15) of the form $\bar{\alpha}$ $=\bar{\alpha}\left(\mathrm{Kn}_{o}, P\right)$ which accounts for the effects of both Knudsen number and pressure ratio. Thus, we have an expression modified from Eq. (17) for large pressure ratios, as

$$
\begin{aligned}
Q= & \frac{\pi r_{0}^{4} p_{o}^{2}}{2 \mu_{0} R T L}\left[\frac{P^{2}-1}{8}+\frac{\mathrm{Kn}_{o}(4+\bar{\alpha})}{4}(P-1)\right. \\
& \left.+(b+\bar{\alpha}) \mathrm{Kn}_{o}^{2} \ln \left(\frac{P-b \mathrm{Kn}_{o}}{1-b \mathrm{Kn}_{o}}\right)\right] .
\end{aligned}
$$

In order to obtain the values of $\bar{\alpha}$ in Eq. (18), we consider a long tube with a large pressure ratio between the inlet and outlet as consisting of many short subtubes connected in series. Each subtube has a small pressure ratio, say, 1.001, between its inlet and outlet, so that the Knudsen number can be assumed to have a fixed value in each subtube and the relationship between pressure drop and mass flow rate in each subtube can be predicted well by Eq. (17). Further, in each subtube in the series, the mass flow rate must be identical, and the inlet pressure of a succeeding subtube should equal the outlet pressure of its upstream neighbor. Finally, the length of the tube should be a sum of the lengths of all subtubes, i.e.,

$$
L=\sum_{j=1}^{n} L_{j},
$$

where $n$ is the total number of subtubes, and $j$ is the sequential number of a subtube starting with the subtube at the outlet.

Rearranging Eq. (18), the length for the entire tube $L$ can be expressed as

$$
\begin{aligned}
L= & \frac{\pi r_{0}^{4} p_{o}^{2}}{2 \mu_{0} R T Q}\left[\frac{P^{2}-1}{8}+\frac{\mathrm{Kn}_{o}(4+\bar{\alpha})}{4}(P-1)\right. \\
& \left.+(b+\bar{\alpha}) \mathrm{Kn}_{o}^{2} \ln \left(\frac{P-b \mathrm{Kn}_{o}}{1-b \mathrm{Kn}_{o}}\right)\right] .
\end{aligned}
$$

Similarly, rearranging Eq. (17), the length for a subtube can be obtained as

$$
\begin{aligned}
L_{j}= & \frac{\pi r_{0}^{4} p_{j}^{2}}{2 \mu_{0} R T Q}\left[\frac{\Delta P^{2}-1}{8}+\frac{\mathrm{Kn}_{j}\left(4+\alpha_{j}\right)}{4}(\Delta P-1)\right. \\
& \left.+\left(b+\alpha_{j}\right) \mathrm{Kn}_{j}^{2} \ln \left(\frac{\Delta P-b \mathrm{Kn}_{j}}{1-b \mathrm{Kn}_{j}}\right)\right],
\end{aligned}
$$

where $\Delta P$ is the pressure ratio between the inlet and outlet of each subtube and $\Delta P=P^{1 / n}, p_{j}=(\Delta P)^{j-1} \cdot p_{o}$ is the outlet pressure of the $j$ th subtube, $\mathrm{Kn}_{j}=\mathrm{Kn}_{o} /(\Delta P)^{j-1}$ is the Knudsen number at the outlet of the subtube, and $\alpha_{j}$ is determined using the mean Knudsen number in the subtube, as

$$
\alpha_{j}=\frac{128}{3 \pi^{2}(1-4 / b)} \tan ^{-1}\left[4.0\left(\frac{2 \mathrm{Kn}_{o}}{(\Delta P+1)(\Delta P)^{j-1}}\right)^{0.4}\right] .
$$

Although Eqs. (20) and (21) appear to be similar, the former includes $\bar{\alpha}$ and may be applied to rarefied gas flows with arbitrary pressure ratio, while the latter is for rarefied gas flows with very small pressure ratio so that a single Knudsen number can be used throughout the tube length. Substituting Eqs. (20) and (21) into Eq. (19), after some mathematical simplification, we obtain the expression for $\bar{\alpha}$ as

$$
\bar{\alpha}=\frac{\left\{\sum_{j=1}^{n}(\Delta P)^{2 j-2}\left[\frac{\Delta P^{2}-1}{8}+\frac{\mathrm{Kn}_{j}\left(4+\alpha_{j}\right)}{4}(\Delta P-1)+\left(b+\alpha_{j}\right) \mathrm{Kn}_{j}^{2} \ln \left(\frac{\Delta P-b \mathrm{Kn}_{j}}{1-b \mathrm{Kn}_{j}}\right)\right]\right\}-\frac{P^{2}-1}{8}-\mathrm{Kn}_{o}(P-1)-b \mathrm{Kn}_{o}^{2} \ln \left(\frac{P-b \mathrm{Kn}_{o}}{1-b \mathrm{Kn}_{o}}\right)}{\frac{\mathrm{Kn}_{o}(P-1)}{4}+\mathrm{Kn}_{o}^{2} \ln \left(\frac{P-b \mathrm{Kn}_{o}}{1-b \mathrm{Kn}_{o}}\right)} .
$$


Equation (22) allows a numerical computation of the value of $\bar{\alpha}$ for different $\mathrm{Kn}_{o}$ and $P$. To use Eq. (22), the number of subtubes should be large enough to render the value of $\bar{\alpha}$ independent of $n$, as shown in Fig. 1. The magnitude of $\bar{\alpha}$ reaches a constant value at a larger number of subtubes as the pressure ratio increases. However, it is clear from Fig. 1 that a value of $n=100$ is adequate for pressure ratios of up to 200 for $\mathrm{Kn}_{o}=0.1$; this is also verified at other values of $\mathrm{Kn}_{o}$ of $1,10,100,1000$, and 10000 . In the following numerical calculations of $\bar{\alpha}, n$ is set at 500. Figure 2 shows the numerically calculated values of $\bar{\alpha}$ for different pressure ratios and Knudsen numbers. These values can be accurately predicted, with a maximum deviation of less than $0.5 \%$, by the correlation

$$
\begin{aligned}
\bar{\alpha}= & \frac{128}{3 \pi^{2}(1-4 / b)} \tan ^{-1} \\
& \times\left[4.0\left(\frac{\mathrm{Kn}_{o}}{\exp \{\exp [1.2271 \ln (\ln (P))-0.6145]\}}\right)^{0.4}\right] .
\end{aligned}
$$

Predictions from this correlation are compared to the actual computed values in Fig. 2. Unlike Eq. (15), Eq. (23) incorporates the influence of pressure ratio and can be applied to gas flows with a significant change in Knudsen number along the length of the tube. As the pressure ratio $P$ approaches 1 , Eq. (23) reduces to Eq. (15), as is also revealed in Fig. 2.

It is now possible to use Eq. (18) to predict the mass flow rate of gas in a tube with large pressure ratios, with $\bar{\alpha}$ determined by Eq. (23). In what follows, the theoretical model specifically refers to these two equations.

Equation (18) may also be used to predict the pressure distribution along the tube. In tube flow, the mass flow rate is constant along the tube length. A part of the flow from any intermediate location $x$, with corresponding pressure $p(x)$, to the outlet can be regarded as an independent flow with its mass flow rate identical to that through the entire tube. We then have

$$
\begin{aligned}
Q= & \frac{\pi r_{0}^{4} p_{o}^{2}}{2 \mu_{0} R T(L-x)}\left[\frac{\bar{p}^{2}-1}{8}+\frac{\mathrm{Kn}_{o}(4+\bar{\alpha})}{4}(\bar{p}-1)\right. \\
& \left.+(b+\bar{\alpha}) \mathrm{Kn}_{o}^{2} \ln \left(\frac{\bar{p}-b \mathrm{Kn}_{o}}{1-b \mathrm{Kn}_{o}}\right)\right],
\end{aligned}
$$

where $\bar{p}=p(x) / p_{o}$ is the normalized pressure at the location $x$. Combining Eqs. (18) and (24) and eliminating $Q$, we obtain

$$
\bar{x}=1-\frac{\bar{p}^{2}-1+2 \mathrm{Kn}_{o}[4+\bar{\alpha}(\bar{p})](\bar{p}-1)+8[b+\bar{\alpha}(\bar{p})] \mathrm{Kn}_{o}^{2} \ln \left(\frac{\bar{p}-b \mathrm{Kn}_{o}}{1-b \mathrm{Kn}_{o}}\right)}{P^{2}-1+2 \mathrm{Kn}_{o}[4+\bar{\alpha}(P)](P-1)+8[b+\bar{\alpha}(P)] \mathrm{Kn}_{o}^{2} \ln \left(\frac{P-b \mathrm{Kn}_{o}}{1-b \mathrm{Kn}_{o}}\right)},
$$

where $\bar{x}=x / L$ is the normalized distance in the flow direction starting from the inlet $(\bar{x}=0)$ and ending at the outlet $(\bar{x}=1)$; $\bar{\alpha}$ in the numerator is calculated in terms of $\bar{p}$ while that in the denominator is in terms of $P$. Equation (25) is an implicit expression for pressure $\bar{p}$ with $\bar{x}$ being the independent variable.

In order to illustrate the improvement in prediction of mass flow rate obtained with the current model, the slip flow model of Beskok et $a .^{26}$ is used here for comparison. The slip flow model treats the gas viscosity as a constant, i.e., $\alpha=0$ in Eq. (14), and uses a first-order slip flow boundary condition, i.e., $b=0$ in Eq. (10). Therefore the expression for mass flow rate for slip flow in a tube is obtained as

$$
Q_{\text {slip }}=\frac{\pi r_{0}^{4} p_{o}^{2}}{2 \mu_{0} R T L}\left[\frac{P^{2}-1}{8}+\operatorname{Kn}_{o}(P-1)\right]
$$

And the implicit dependence of the slip flow pressure $\bar{p}_{\text {slip }}$ $=p(x)_{\text {slip }} / p_{o}$ on distance along the tube is written as

$$
\bar{x}=1-\frac{\bar{p}_{\text {slip }}^{2}-1+8 \mathrm{Kn}_{o}(\bar{p}-1)}{P^{2}-1+8 \mathrm{Kn}_{o}(P-1)} .
$$

From Eq. (27), an explicit expression for $\bar{p}_{\text {slip }}$ can be deduced as

$$
\bar{p}_{\text {slip }}(\bar{x})=-4 \mathrm{Kn}_{o}+\sqrt{\left(4 \mathrm{Kn}_{o}\right)^{2}+\left[8 \mathrm{Kn}_{o}(1-P)+1-P^{2}\right] \bar{x}+P\left(P+8 \mathrm{Kn}_{o}\right)} .
$$




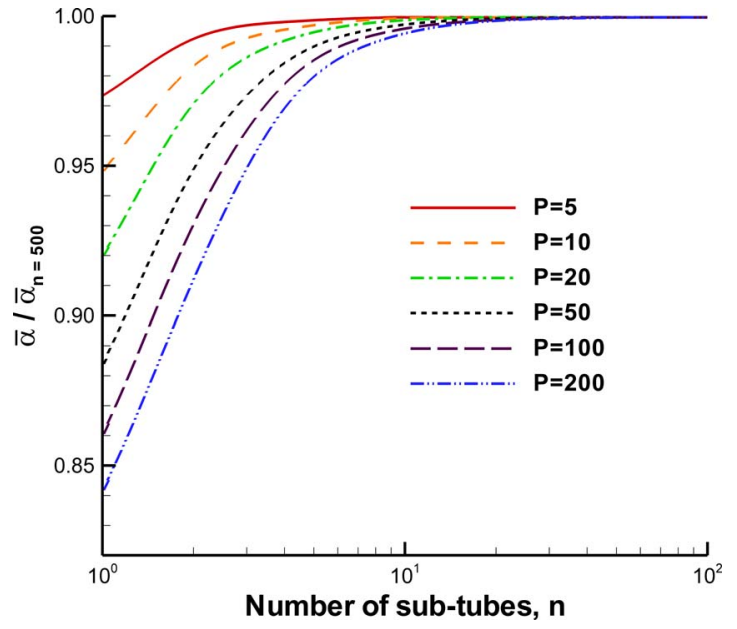

FIG. 1. (Color online) Influence of number of subtubes on $\bar{\alpha}\left(\mathrm{Kn}_{o}=0.1\right)$.

Predictions from the model developed in this work and the experimental data will be compared to the slip flow model in Eqs. (26) and (28) later in this discussion.

\section{EXPERIMENTAL STUDY}

\section{A. Experimental setup}

To validate the model developed in this work, an experimental study was conducted on rarefied gas flow through microtubes over a range of Knudsen numbers and pressure ratios. Figure 3 shows a schematic illustration of the experimental setup designed for this purpose.

The microtubes being tested were fused silica capillary tubes (Polymicro Technology ${ }^{\mathrm{TM}}$ ) with inner diameters of 20, 50 , and $100 \mu \mathrm{m}$ and root mean square roughness on the inner surface of less than $5 \mathrm{~nm}$. The inlet of the capillary tube was connected to an enclosed gas reservoir while its outlet was connected to a vacuum chamber. Temperatures of

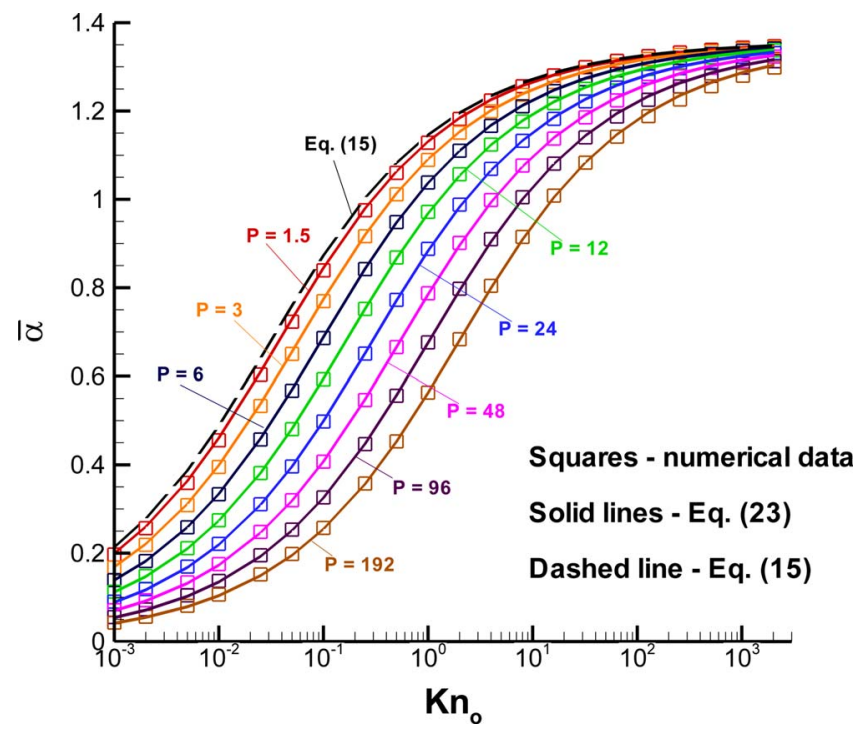

FIG. 2. (Color online) Computed values of $\bar{\alpha}$ at different outlet Knudsen numbers and pressure ratios; also shown are predictions from the current model, Eq. (23), as well as from the literature, Eq. (15).

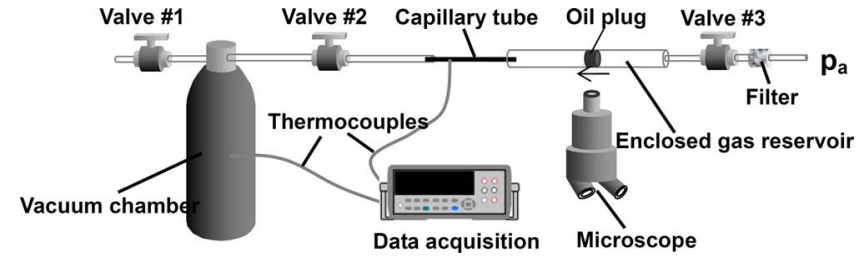

FIG. 3. (Color online) Schematic illustration of experimental setup.

the vacuum chamber and the capillary tube were monitored using thermocouples (Omega) attached to the outside walls and recorded using a multimeter (Agilent 34410A). Three valves were used to control the flow path of the air, which is the gas used in the tests.

\section{B. Experimental procedures}

During a test, valve 3 was first opened to the atmosphere, so that air flowed into the enclosed gas reservoir and the pressure inside the reservoir reached atmosphere pressure $P_{a}$. A nonvolatile oil plug (soybean oil) was then introduced into the gas reservoir to partition the reservoir into two sections, as shown in Fig. 3. At this time, valve 3 was closed. The vacuum chamber filled with de-ionized water was heated up to the boiling point of water. The water vapor generated in the process helped expel the air in the chamber through valve 1 , with valve 2 remaining closed. When the air was completely expelled, valve 1 was closed. Complete expulsion of air was judged to have occurred when the volumetric ratio of the air that was initially present in the chamber to the water vapor fell below $0.01 \%$; the vapor volume for this calculation was estimated from the reduction in volume of the remaining water in the vacuum chamber. This procedure leads to only water and its vapor being present in the vacuum chamber, so that the pressure inside the chamber is equal to the saturation pressure of water. The desired steady temperature was achieved in the vacuum chamber by allowing it to equilibrate with the surroundings for over $1 \mathrm{~h}$ for room temperature or by embedding the chamber in deionized ice water when a lower chamber temperature was needed. The vacuum chamber temperature was measured using an attached thermocouple. The saturated pressure inside the vacuum chamber was then calculated using the GoffGratch equation. ${ }^{27}$

Valve 2 was then opened and the air previously stored in the enclosed gas reservoir flowed through the capillary tube toward the vacuum chamber, driven by the pressure drop between the inlet and outlet of the capillary tube. As the air flowed out of the gas reservoir into the vacuum chamber, the inlet pressure to the capillary tube gradually dropped and pulled the oil plug from right to left. The movement of the left-side meniscus of the oil plug was tracked with a traveling microscope. The position of the right-side meniscus of the oil plug was also recorded as a function of time for later calculations.

It may be noted that although the air flow inside the capillary tube may appear to be an unsteady process due to the continuous decrease in the inlet pressure, it may readily 
TABLE I. Test cases.

\begin{tabular}{lccccccc}
\hline \hline Case & $\begin{array}{c}r_{0} \\
(\mu \mathrm{m})\end{array}$ & $\begin{array}{c}L \\
(\mathrm{~mm})\end{array}$ & $\begin{array}{c}p_{a} \\
(\mathrm{~Pa})\end{array}$ & $\begin{array}{c}p_{o} \\
(\mathrm{~Pa})\end{array}$ & $\mathrm{Kn}_{o}$ & $\begin{array}{c}T \\
(\mathrm{~K})\end{array}$ & $\begin{array}{c}\mu_{0} \\
\left(10^{-5} \mathrm{~kg} \mathrm{~m}^{-1} \mathrm{~s}^{-1}\right)\end{array}$ \\
\hline $\mathrm{A}$ & 50 & 95 & 101325 & $2337^{\mathrm{a}}$ & 0.0564 & 293 & 1.82 \\
$\mathrm{~B}$ & 25 & 61 & 101325 & $2337^{\mathrm{a}}$ & 0.113 & 293 & 1.82 \\
$\mathrm{C}$ & 10 & 33 & 101325 & $2337^{\mathrm{a}}$ & 0.282 & 293 & 1.82 \\
$\mathrm{D}$ & 10 & 27 & 101325 & $608^{\mathrm{b}}$ & 1.08 & 293 & 1.82 \\
\hline
\end{tabular}

${ }^{\mathrm{a}}$ The temperature of the vacuum chamber is $20.5^{\circ} \mathrm{C}$

${ }^{\mathrm{b}}$ The temperature of the vacuum chamber is $0{ }^{\circ} \mathrm{C}$.

be treated as a quasisteady flow, based on the Strouhal number $\mathrm{Sr}=L / u_{c} t_{c}$ of the flow where $u_{c}$ and $t_{c}$ are the characteristic velocity and time. In all the tests in this work, the slowest air velocity in the capillary tube is approximately $10 \mathrm{~m} / \mathrm{s}$ and the longest capillary tube is $100 \mathrm{~mm}$ in length. It takes at least $30 \mathrm{~s}$ for $l_{1}$ to increase by $1 \%$, or in other words, for $p_{i}$ to decrease by $1 \%$ according to Eq. (29). Using these parameters, the largest possible Strouhal number is estimated to be 0.00033 . Any unsteady effects caused at this very small Strouhal number are negligible, so that the gas flow in the present work can be considered to be steady.

The pressure at the outlet of the capillary tube is assumed to be the saturation pressure of vapor (equal to the value in the vacuum chamber). The gradual flow of air from the gas reservoir into the vapor chamber could cause some error in this assumption. However, the influence of the added air on the pressure in the vacuum chamber, estimated by considering the ratio of volumes of the gas reservoir and the vacuum chamber, is negligibly small, since this volumetric ratio is only $0.03 \%$. Even if all the air in the gas reservoir were added to the vacuum chamber, the error caused by the added air to the vacuum chamber pressure would still be negligible.

\section{Parametric measurements}

The inlet pressure $p_{i}$ is deduced from the length $l_{1}$ of the right-side part of the air column in the gas reservoir. The length $l_{1}$ was determined from the recorded positions of the right-side meniscus at different times. Since the original pressure in the gas reservoir is atmospheric pressure $p_{a}, p_{i}$ is determined by

$$
p_{i}=p_{a} l_{1}^{(0)} / l_{1},
$$

where $l_{1}^{(0)}$ is the initial length of the right-side air column. The mass flow rate through the capillary tube can be derived from the rate of decrease in mass in the gas reservoir using the following expression:

$$
Q=\frac{\pi d^{2}\left(l_{1}^{(0)}+l_{2}^{(0)}\right)}{4 R T} \frac{d p_{i}}{d t}=-\frac{\pi d^{2}\left(l_{1}^{(0)}+l_{2}^{(0)}\right) p_{a} l_{1}^{(0)}}{4 R T l_{1}^{2}} \frac{d l_{1}}{d t}
$$

where $l_{2}^{(0)}$ is the initial length of the left-side air column, and $d$ the inner diameter of the gas reservoir. The rate of change in $l_{1}$, i.e., $d l_{1} / d t$, is computed over a short time interval as $\Delta l_{1} / \Delta t$, where $\Delta l_{1}=0.002 \mathrm{~m}$ and $\Delta t$ is the time interval for the meniscus to travel this distance. Since $0.002 \mathrm{~m}$ is very small compared with the total travel distance $(0.2 \mathrm{~m})$ of the meniscus and the observed velocity of the meniscus changes very little over this distance, this is an acceptable estimate of the average velocity. The pressure difference to drive the oil plug was estimated by placing an oil plug in a tube held at different inclinations, and the observed critical inclination for the oil plug to start moving was used to calculate the flow resistance. The maximum flow resistance among all the tubes used in the current experiments was found to be approximately $50 \mathrm{~Pa}$ for a gas-reservoir tube of inner diameter of 2 $\mathrm{mm}$, which is much smaller than the absolute pressure inside the gas reservoir $\left(1 \times 10^{4}-1 \times 10^{5} \mathrm{~Pa}\right)$. Therefore, its effect on the calculation of mass flow rate was neglected.

The outlet pressure in the experiments is equal to the saturation vapor pressure $p_{s}$ inside the vacuum chamber and is determined by the Goff-Gratch equation, ${ }^{27}$

$$
\begin{aligned}
\log _{10} p_{s}= & -7.90298(373.16 / T-1) \\
& +5.02808 \log _{10}(373.16 / T) \\
& -1.3816 \times 10^{-7}\left(10^{11.344(1-T / 373.16)}-1\right) \\
& +8.1328 \times 10^{-3}\left(10^{-3.49149(373.16 / T-1)}-1\right) \\
& +\log _{10}(101324.6),
\end{aligned}
$$

where $T$ is in kelvin. This equation is valid over a temperature range of $\left[-50{ }^{\circ} \mathrm{C}, 102{ }^{\circ} \mathrm{C}\right]$ and has a deviation of less than $0.1 \%$ from the experimental data in the temperature range of $\left[0{ }^{\circ} \mathrm{C}, 30{ }^{\circ} \mathrm{C}\right]$ relevant to the current study.

Knowing $p_{s}$, the Knudsen number at the outlet of the capillary tubes is calculated as

$$
\mathrm{Kn}_{o}=\frac{16 \mu_{0}}{5 \sqrt{2 \pi R T} \rho_{o} r_{0}}=\frac{16 \mu_{0} \sqrt{R T}}{5 \sqrt{2 \pi} p_{o} r_{0}}
$$

\section{Test cases}

Tests were carried out for air flow with Knudsen number in the range $0.05<\mathrm{Kn}_{o}<1$, i.e., mainly in the transition flow regime, as listed in Table $\mathrm{I}$. 


\section{E. Uncertainty analysis}

Based on Eq. (30), the uncertainty in the measured mass flow rate, denoted by the subscript $m$, may be estimated as

$$
\begin{aligned}
& \left(\frac{\Delta Q}{Q}\right)_{m} \\
& \quad=\sqrt{4\left(\frac{\Delta d}{d}\right)^{2}+\left(\frac{2 l_{1}^{(0)}+l_{2}^{(0)}}{l_{1}^{(0)}+l_{2}^{(0)}}\right)^{2}\left(\frac{\Delta l_{1}^{(0)}}{l_{1}^{(0)}}\right)^{2}+\left(\frac{l_{2}^{(0)}}{l_{1}^{(0)}+l_{2}^{(0)}}\right)^{2}\left(\frac{\Delta l_{2}^{(0)}}{l_{2}^{(0)}}\right)^{2}+\left(\frac{\Delta\left(\Delta l_{1}\right)}{\Delta l_{1}}\right)^{2}+\left(\frac{\Delta(\Delta t)}{\Delta t}\right)^{2}+\left(\frac{\Delta T}{T}\right)^{2}+4\left(\frac{\Delta l_{1}}{l_{1}}\right)^{2}+\left(\frac{\Delta p_{a}}{p_{a}}\right)^{2}} .
\end{aligned}
$$

Since the calculated mass flow rate calculated using Eq. (18) also depends on experimental parameters such as $r_{0}$ and $p_{o}$, the uncertainty in this flow rate, denoted by the subscript $c$, may be estimated as

$$
\begin{aligned}
& \left(\frac{\Delta Q}{Q}\right)_{c}=\left\{16\left(\frac{\Delta r_{0}}{r_{0}}\right)^{2}+4\left(\frac{\Delta p_{o}}{p_{o}}\right)^{2}+\left(\frac{\Delta \mu}{\mu}\right)^{2}+\left(\frac{\Delta T}{T}\right)^{2}+\left(\frac{\Delta L}{L}\right)^{2}\right. \\
& +P^{2}\left[\frac{2 P+2 \mathrm{Kn}_{o}(4+\bar{\alpha})+8(b+\bar{\alpha}) \mathrm{Kn}_{o}^{2} /\left(P-b \mathrm{Kn}_{o}\right)}{P^{2}-1+2 \mathrm{Kn}_{o}(4+\bar{\alpha})(P-1)+8(b+\bar{\alpha}) \mathrm{Kn}_{o}^{2} \ln \left(\frac{P-b \mathrm{Kn}_{o}}{1-b \mathrm{Kn}_{o}}\right)}\right]^{2}\left(\frac{\Delta P}{P}\right)^{2}+\mathrm{Kn}_{o}^{2}\left(\frac{\Delta \mathrm{Kn}_{o}}{\mathrm{Kn}_{o}}\right)^{2} \\
& \times\left[\frac{(8+2 \bar{\alpha})(P-1)+16 \mathrm{Kn}_{o}(b+\bar{\alpha}) \ln \left(\frac{P-b \mathrm{Kn}_{o}}{1-b \mathrm{Kn}_{o}}\right)+8 \mathrm{Kn}_{o}^{2}(b+\bar{\alpha}) b \frac{P-1}{\left(P-b \mathrm{Kn}_{o}\right)\left(1-b \mathrm{Kn}_{o}\right)}}{P^{2}-1+2 \mathrm{Kn}_{o}(4+\bar{\alpha})(P-1)+8(b+\bar{\alpha}) \mathrm{Kn}_{o}^{2} \ln \left(\frac{P-b \mathrm{Kn}_{o}}{1-b \mathrm{Kn}_{o}}\right)}\right]^{2} \\
& \left.+\left[\frac{2 \mathrm{Kn}_{o}(P-1)+8 \mathrm{Kn}_{o}^{2} \ln \left(\frac{P-b \mathrm{Kn}_{o}}{1-b \mathrm{Kn}_{o}}\right)}{P^{2}-1+2 \mathrm{Kn}_{o}(4+\bar{\alpha})(P-1)+8(b+\bar{\alpha}) \mathrm{Kn}_{o}^{2} \ln \left(\frac{P-b \mathrm{Kn}_{o}}{1-b \mathrm{Kn}_{o}}\right)}\right] \bar{\alpha}^{2}\left(\frac{\Delta \bar{\alpha}}{\bar{\alpha}}\right)^{2}\right\}^{0.5},
\end{aligned}
$$

where

$$
\left(\frac{\Delta P}{P}\right)=\sqrt{\left(\frac{\Delta p_{a}}{p_{a}}\right)^{2}+\left(\frac{\Delta l_{1}^{(0)}}{l_{1}^{(0)}}\right)^{2}+\left(\frac{\Delta l_{1}}{l_{1}}\right)^{2}+\left(\frac{\Delta p_{o}}{p_{o}}\right)^{2}}
$$

and

$$
\left(\frac{\Delta \mathrm{Kn}_{o}}{\mathrm{Kn}_{o}}\right)=\sqrt{\left(\frac{\Delta \mu_{a}}{\mu_{a}}\right)^{2}+\frac{1}{4}\left(\frac{\Delta T}{T}\right)^{2}+\left(\frac{\Delta p_{o}}{p_{o}}\right)^{2}+\left(\frac{\Delta r_{0}}{r_{0}}\right)^{2}} .
$$

The relative uncertainty in $\Delta \bar{\alpha} / \bar{\alpha}$ in Eq. (33) depends on $\Delta P / P$ and $\Delta \mathrm{Kn}_{o} / \mathrm{Kn}_{o}$ and is calculated using a similar approach but the related expressions are not listed here in the interest of brevity.

Finally, the total relative uncertainty in the mass flow rate can be written as

$$
\frac{\Delta Q}{Q}=\sqrt{\left(\frac{\Delta Q}{Q}\right)_{m}^{2}+\left(\frac{\Delta Q}{Q}\right)_{c}^{2}} .
$$

The relative uncertainties in all the measured variables are listed in Table II. Using the values in Table II, the total uncertainty in the mass flow rates reported in this work is estimated using Eq. (34) to be

$$
\frac{\Delta Q}{Q}< \pm 5.5 \%
$$

\section{RESULTS AND DISCUSSION}

\section{A. Mass flow rate}

The mass flow rates through the four different microtubes, measured at different pressure ratios and Knudsen numbers, are shown in Fig. 4; predictions from Eq. (18) are also included in the figure. The maximum pressure ratio in the experiments was approximately 170 (case D), with the inlet and outlet pressures being 101325 and $608 \mathrm{~Pa}$, respectively. In each case, mass flow rate increases with pressure ratio, and the theoretical predictions are seen to agree well with the experimental results. The evident nonlinear relationship between $Q$ and $P$ shows the important influence of air compressibility on flow behavior.

The theoretical model developed here is also compared to the slip and nonslip models from the literature. This comparison is facilitated by normalizing the mass flow rates against those predicted by the slip flow model as follows: 


$$
R_{1}=\frac{Q}{Q_{\text {slip }}}=\frac{P^{2}-1+\mathrm{Kn}_{o}(8+2 \bar{\alpha})(P-1)+8(b+\bar{\alpha}) \mathrm{Kn}_{o}^{2} \ln \left(\frac{P-b \mathrm{Kn}_{o}}{1-b \mathrm{Kn}_{o}}\right)}{P^{2}-1+8 \mathrm{Kn}_{o}(P-1)}
$$

and

$$
R_{2}=\frac{Q_{\text {nonslip }}}{Q_{\text {slip }}}=\frac{P^{2}-1}{P^{2}-1+8 \mathrm{Kn}_{o}(P-1)} .
$$

$R_{1}$ is the ratio of the mass flow rates obtained with the current model and the slip flow model, while $R_{2}$ is that between the nonslip flow model and the slip flow model. Figure 5 shows values of $R_{1}$ and $R_{2}$ at different pressure ratios for three different Knudsen numbers. The experimental results (obtained by dividing the experimental mass flow rate with that from the slip flow model) are also plotted in Fig. 5, showing good agreement with the curve for $R_{1}$ (the theoretical model from the present work), which clearly demonstrates the improvements obtained from the current model relative to the slip flow model for rarefied gas flows in the transitional regime. The improvements in the current theoretical model are achieved by considering both the secondorder slip boundary equation (10) and rarefaction of the viscosity equation (14), which are applicable for all flow regimes. ${ }^{25}$ The experimental data exceed the theoretical predictions at small pressure ratio in Fig. 5(a), which may be caused by small amounts of air leakage from the atmosphere into the gas reservoir. As the pressure ratio becomes small, the inlet pressure inside the gas reservoir approaches that of the vacuum chamber, with an increased chance of leakage from the surrounding air. Further, the ratio $R_{1}$ is clearly larger than unity, especially at small $P$ and large $\mathrm{Kn}_{o}$, implying the occurrence of a higher flow rate compared to the slip flow model prediction. As $P$ becomes large, $R_{1}$ approaches unity for each $\mathrm{Kn}_{o}$ in Fig. 5. This is because as the pressure increases, air flow in much of the tube falls in the slip flow regime. The ratio $R_{2}$ is lower than unity, especially at small $P$ (near-constant Knudsen flows) and large $\mathrm{Kn}_{o}$. This indicates that the flow rate of the nonslip flow model is significantly smaller than that of the slip flow model when gas flow in the entire tube is at a high Knudsen number. As $P$ becomes large, $R_{2}$ approaches unity at each $\mathrm{Kn}_{o}$ in Fig. 5. This is because at large $P$, gas flow in much of the tube has a low local Knudsen number, despite $\mathrm{Kn}_{o}$ at the outlet being high, and continuum flow with nonslip boundary condition dominates the overall flow rate in the tube; this causes the differ- ence between the slip flow model and the nonslip flow model to be small at large $P$.

The observation that $R_{2}$ falls well below $R_{1}$ indicates that rarefaction effects, including slip at the wall and a decrease in the viscosity, are important for the cases studied here.

The influence of the Knudsen number on rarefied gas flows can be shown by observing its effect on the variation of the mass flow rate normalized against that in a free molecular flow $Q_{\text {free }}$ defined as

$$
Q_{\text {free }}=\frac{4}{3} r_{0}^{3} \frac{\Delta p}{L}\left(\frac{2 \pi}{R T}\right)^{0.5},
$$

where $\Delta p$ is the pressure drop between the inlet and outlet of the capillary tube. Figure 6 shows the dependence of normalized mass flow rate on $\mathrm{Kn}_{o}$. As seen, three flow regimes, i.e., slip $\left(0.001<\mathrm{Kn}_{o}<0.1\right)$, transition $\left(0.1<\mathrm{Kn}_{o}<10\right)$, and free molecular $\left(\mathrm{Kn}_{o}>10\right)$ flows, are covered. When $\mathrm{Kn}_{o}$ becomes large, the air flow gradually turns into a free molecular flow, so that the normalized flow rate approaches unity. The experimental data are mainly in the transition flow regime $\left(0.1<\mathrm{Kn}_{o}<10\right)$ and agree well with the theoretical predictions. It is evident that pressure ratio $P$ plays an important role on mass flow rate. As $P$ becomes large, the entire curve shifts from left to right. Loyalka and Hamoodi's solutions of the linearized Boltzmann equation ${ }^{20}$ are also plotted in the figure to compare the measured gas flow rates at an extremely small pressure ratio of $P=1.001$, for which Knudsen number is almost constant throughout the tube. Although experimental data could only be obtained over some of the flow regimes, the good agreement between the solution of the linear-Boltzmann equation and the curve for $P=1.001 \mathrm{im}-$ plies that Eq. (18) is applicable for flows over a wide range of Knudsen numbers; this is also evidenced by the fact that Eq. (18) predicts a mass flow rate which is identical to that of free molecular flow, i.e., the normalized flow rate becomes unity as $\mathrm{Kn}_{o}$ approaches infinity in Fig. 6 .

The inset in Fig. 6 shows details of the flow rate in the transition flow regime. As a comparison, the results predicted by the slip flow model (dashed lines) are also presented. It is

TABLE II. Relative uncertainties in the measured variables.

\begin{tabular}{lccccccc}
\hline \hline Measured variables $A$ & $l_{1}$ & $l_{2}$ & $d$ & $T$ & $p_{o}$ & $r_{0}$ & $L$ \\
\hline Relative uncertainty $(\Delta A / A)(\%)$ & 0.5 & 0.05 & 0.5 & 0.05 & 1.0 & 0.25 & 1.0 \\
Measured variables $A$ & $l_{1}^{(0)}$ & $l_{2}^{(0)}$ & $\bar{\alpha}$ & $\Delta l_{1}$ & $\Delta t$ & $\mu$ & $p_{a}$ \\
\hline Relative uncertainty $(\Delta A / A)(\%)$ & 0.5 & 0.05 & 0.5 & 1.0 & 0.01 & 1.0 & 0.1 \\
\hline \hline
\end{tabular}



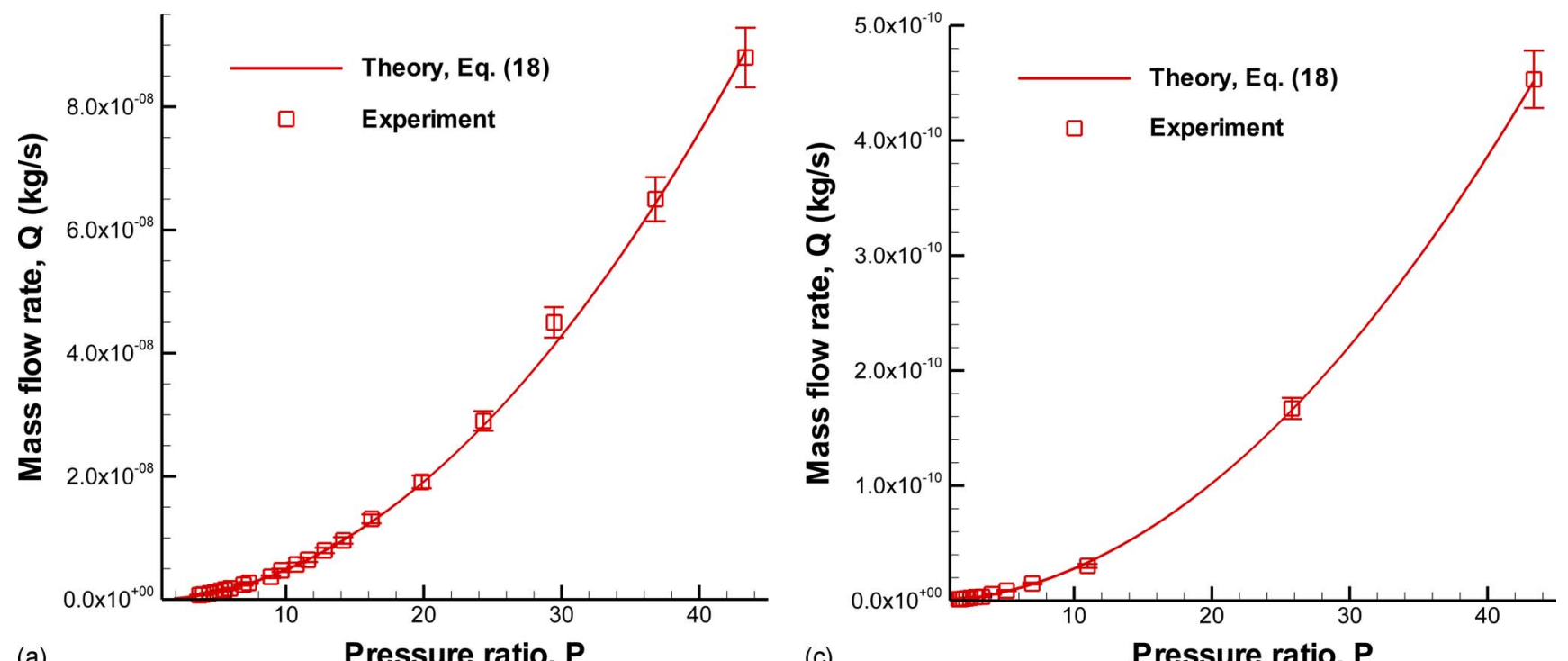

(a)

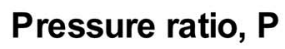

(c)
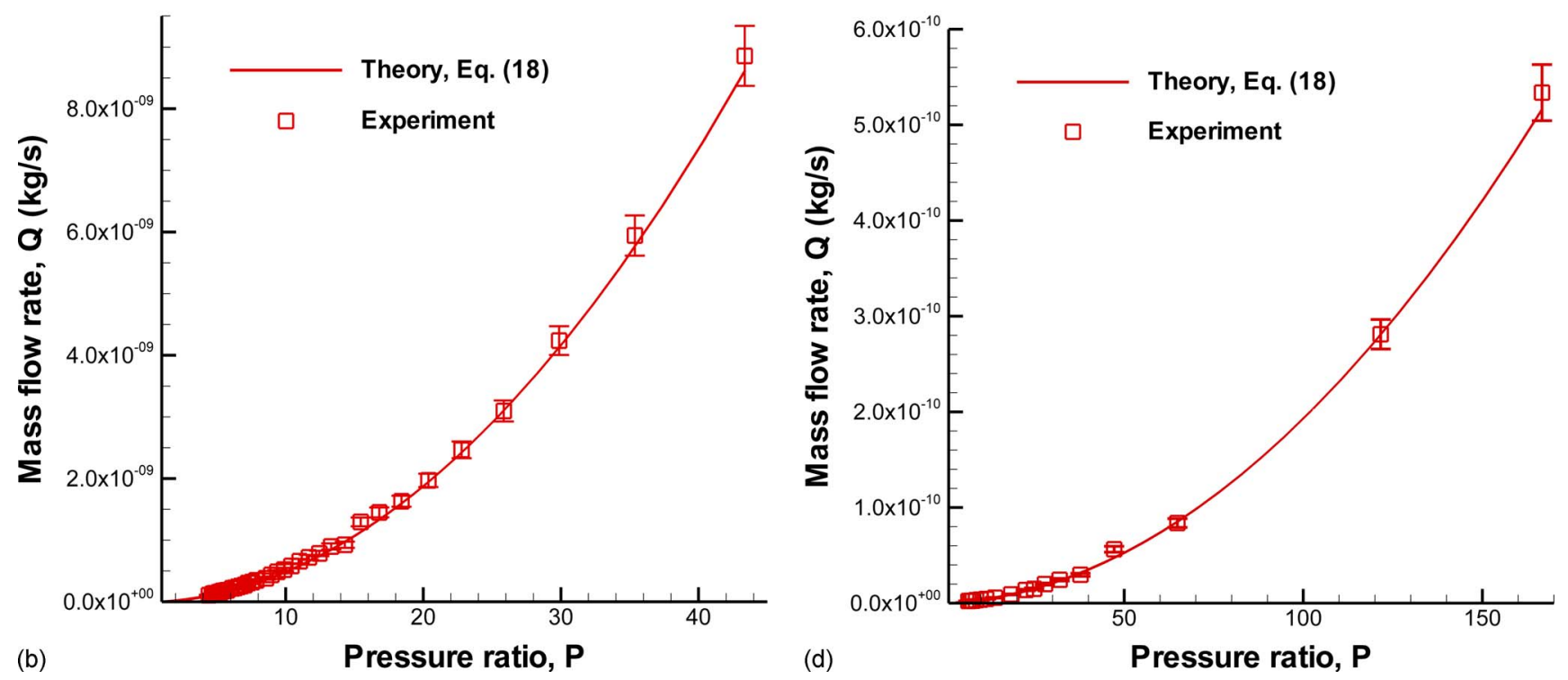

FIG. 4. (Color online) Mass flow rate $Q$ vs pressure ratio $P$ : (a) case A, (b) case B, (c) case C, and (d) case D. Descriptions of the cases are available in Table I.

evident that the current model predicts the experimental data much better than does the slip flow model, especially for flows at low pressure ratios, e.g., $P=3$ and 6 , and at high Knudsen numbers, e.g., $\mathrm{Kn}_{o}=1.08$. We further note that the current model is able to resolve the expected dip in mass flow rate below the value of $Q / Q_{\text {free }}=1$ that would be obtained in free molecular flow. In the inset, the Knudsen number at which the normalized flow rate is a minimum is seen to change its position with pressure ratio $P$.

The minimum in the normalized flow rate and the corresponding Knudsen number can be calculated for air flows at different pressure ratios using Eqs. (18) and (35). Figure 7 shows that the minimum normalized flow rate and the corresponding Knudsen number generally increase with pressure ratio. When $P$ is close to unity (i.e., a gas flow with the Knudsen number nearly unchanged along the tube length), the corresponding Knudsen number is approximately 3, and the Knudsen's minimum is 0.915 ; this is consistent with re- sults reported in the literature ${ }^{20,25}$ for these lower pressure ratios.

\section{B. Streamwise pressure distribution}

Measuring streamwise pressure distribution in microtubes is experimentally challenging. Although pressure sensors were integrated into a microchannel in one study, ${ }^{22}$ extra channels were needed to transfer gas from the microchannels to the sensors in this case, which may have caused disturbance in the flow. Existing fabrication techniques for pressure measurement are also more adapted to flat channels.

In this section, we deduce the streamwise pressure distribution from the available experimental data for mass flow rate. The derivation is based on the fact that mass flow rate is inversely proportional to tube length, as seen in Eq. (18). Additional experiments were also conducted to verify this behavior in the current channels, as shown in the log-log plot 

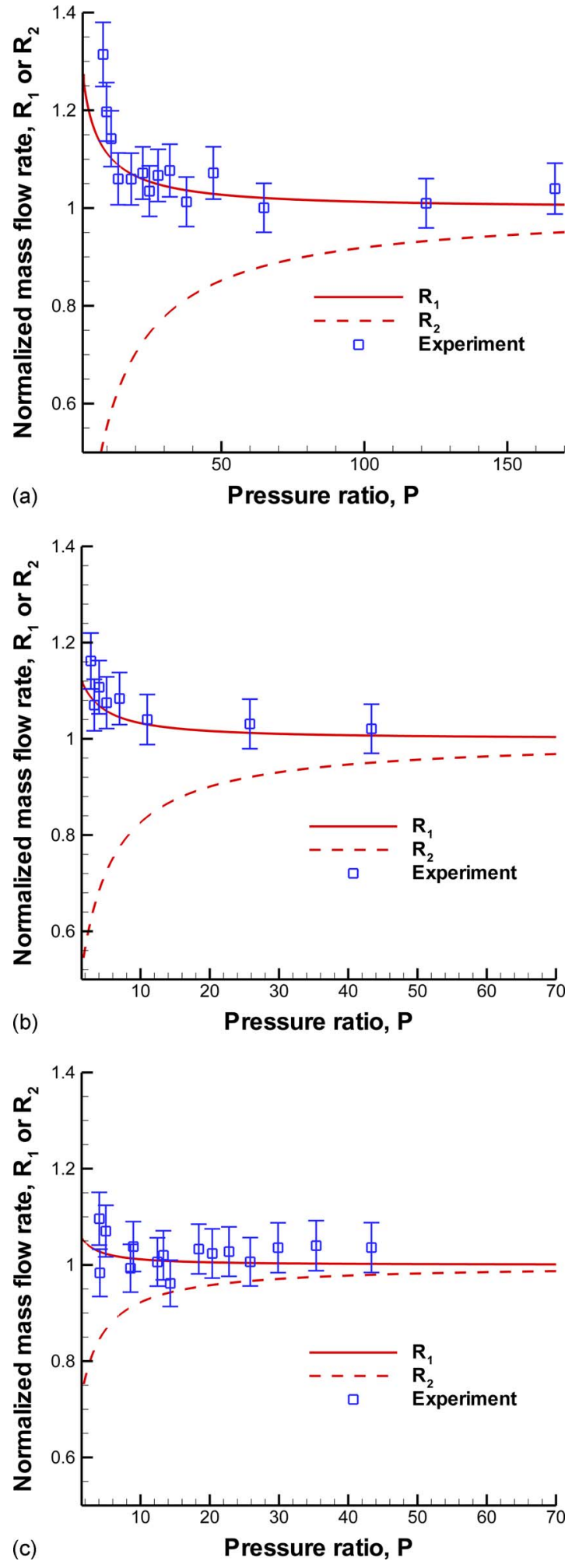

FIG. 5. (Color online) Variation of normalized mass flow rates $R_{1}$ and $R_{2}$ with pressure ratio $P$ : (a) $\mathrm{Kn}_{o}=1.08$, (b) $\mathrm{Kn}_{o}=0.282$, and (c) $\mathrm{Kn}_{o}=0.113$.

of Fig. 8. Both the experimental and theoretical data exhibit a precise inverse proportionality between mass flow rate and tube length, with slopes of -1 for the curves.

According to Eq. (18) and the experimental results in Fig. 8, $Q$ is inversely proportional to $L$, and this relationship can be expressed as

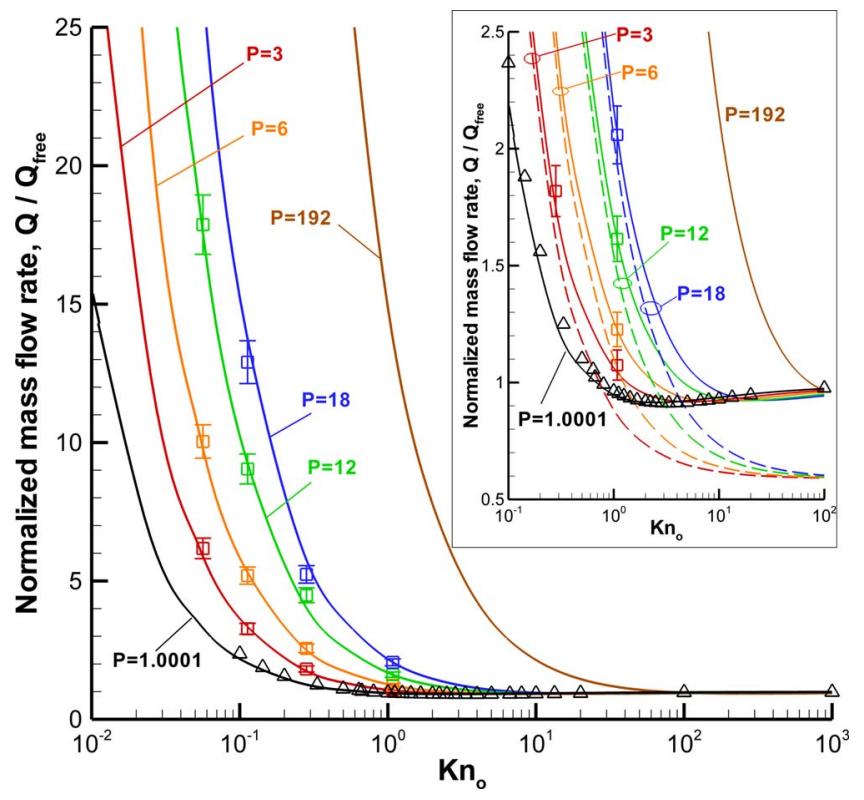

FIG. 6. (Color online) Influence of the outlet Knudsen number $\mathrm{Kn}_{o}$ on mass flow rate at different pressure ratios: solid line, current model; dashed line, slip flow model; $\square$, experimental data; $\triangle$, solution of the linear-Boltzmann equations by Loyalka and Hamoodi (Ref. 20).

$$
Q L=C\left(P, \mathrm{Kn}_{o}, r_{0}, p_{o}, T\right),
$$

where $C$ is a function of parameters $P, \mathrm{Kn}_{o}, r_{0}, p_{o}$, and $T$. In a tube of length $L_{0}$ with a mass flow rate of $Q_{0}$, the normalized pressure $\bar{p}$ gradually drops from $P$ at the inlet to 1 at the outlet. A part of this tube flow which starts at an arbitrary streamwise location $x\left(0<x<L_{0}\right)$ and ends at the outlet $(x$ $=L_{0}$ ) can be treated independently, with the inlet flow pressure being $\bar{p}(x)$ such that Eq. (36) becomes

$$
Q_{0} L_{x}\left(\bar{p}, \mathrm{Kn}_{o}, r_{0}, p_{o}, T\right)=C\left(\bar{p}, \mathrm{Kn}_{o}, r_{0}, p_{o}, T\right),
$$

where $L_{x}$ is the distance from the location of $x$ to the outlet, i.e., $L_{x}=L_{0}-x$. The mass flow rate $Q_{0}$ is a constant for the

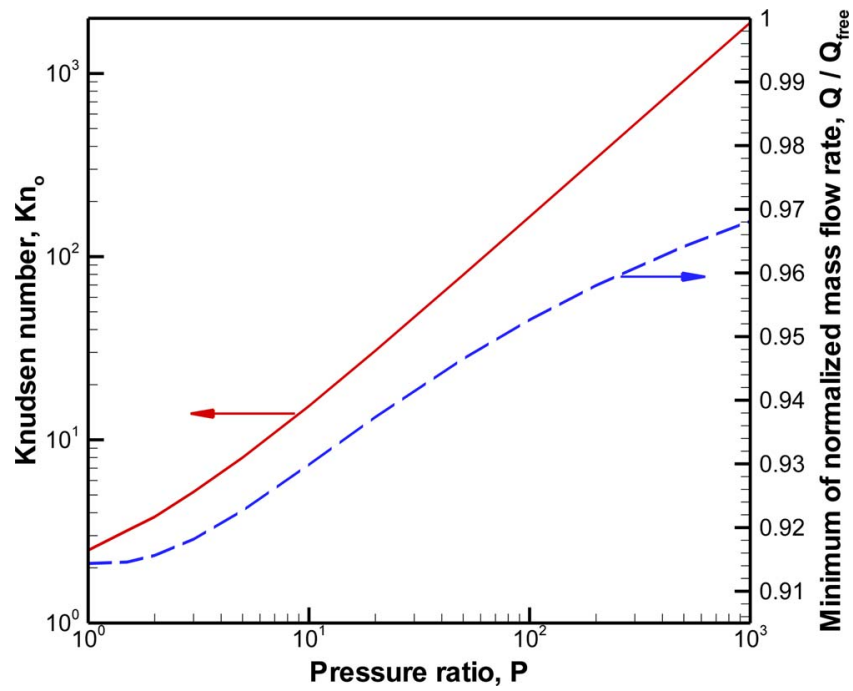

FIG. 7. (Color online) Minimum in the normalized mass flow rate (dashed line) and the corresponding Knudsen number (solid line) at different pressure ratios. 


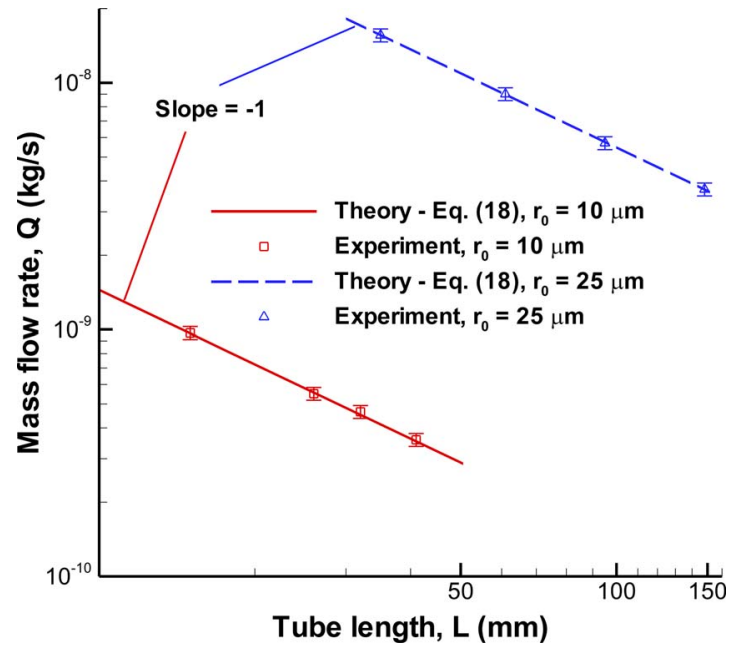

FIG. 8. (Color online) Influence of tube length on mass flow rate for two different tube radii $\left(P=43, \mathrm{Kn}_{o}=0.282\right)$.

flow and $L_{x}$ is a function of parameters $\bar{p}, \mathrm{Kn}_{o}, r_{0}, p_{o}$, and $T$. The right side of Eq. (37) may be further rewritten as

$$
\begin{aligned}
C\left(\bar{p}, \mathrm{Kn}_{o}, r_{0}, p_{o}, T\right) & =C\left(P, \mathrm{Kn}_{o}, r_{0}, p_{o}, T\right) \\
& =Q\left(P, \mathrm{Kn}_{o}, r_{0}, p_{o}, T\right) L,
\end{aligned}
$$

where $P=\bar{p}$, and $L$, a constant, is the length of a tube. Based on Eqs. (37) and (38), it is easy to obtain the equivalency

$$
Q_{0} L_{x}\left(\bar{p}, \mathrm{Kn}_{o}, r_{0}, p_{o}, T\right)=Q\left(P, \mathrm{Kn}_{o}, r_{0}, p_{o}, T\right) L
$$

and

$$
L_{x}\left(\bar{p}, \mathrm{Kn}_{o}, r_{0}, p_{o}, T\right)=\frac{Q\left(P, \mathrm{Kn}_{o}, r_{0}, p_{o}, T\right) L}{Q_{0}} .
$$

Equation (40) shows that the distance $L_{x}$ at which the pressure is $\bar{p}$ for a mass flow rate of $Q_{0}$ may instead be calculated from a different situation with the same $\mathrm{Kn}_{o}, r_{0}, p_{o}$, and $T$ but with a mass flow rate of $Q$ and a pressure ratio of $P$ $(P=\bar{p})$. According to Eq. (40), the distance $L_{0}$ at which $\bar{p}$ $=P_{\max }$ can be calculated by simply substituting $P$ with $P_{\max }$, i.e.,

$$
L_{0}=\frac{Q\left(P_{\max }, \mathrm{Kn}_{o}, r_{0}, p_{o}, T\right) L}{Q_{0}} .
$$

Normalizing $L_{x}$ using $L_{0}$, we have

$$
\begin{aligned}
\frac{L_{x}\left(\bar{p}, \mathrm{Kn}_{o}, r_{0}, p_{o}, T\right)}{L_{0}} & =\frac{L_{0}-x\left(\bar{p}, \mathrm{Kn}_{o}, r_{0}, p_{o}, T\right)}{L_{0}} \\
& =\frac{Q\left(P, \mathrm{Kn}_{o}, r_{0}, p_{o}, T\right)}{Q\left(P_{\max }, \mathrm{Kn}_{o}, r_{0}, p_{o}, T\right)} .
\end{aligned}
$$

Finally, the normalized flow distance at which $\bar{p}=P$ may be expressed as

$$
\bar{x}\left(\bar{p}, \mathrm{Kn}_{o}, r_{0}, p_{o}, T\right)=1-\frac{Q\left(P, \mathrm{Kn}_{o}, r_{0}, p_{o}, T\right)}{Q\left(P_{\max }, \mathrm{Kn}_{o}, r_{0}, p_{o}, T\right)} .
$$

The flow rate $Q$ in the numerator in Eq. (43) is experimentally obtained at different pressure ratios in each case in Table I, while that in the denominator is the flow rate at the

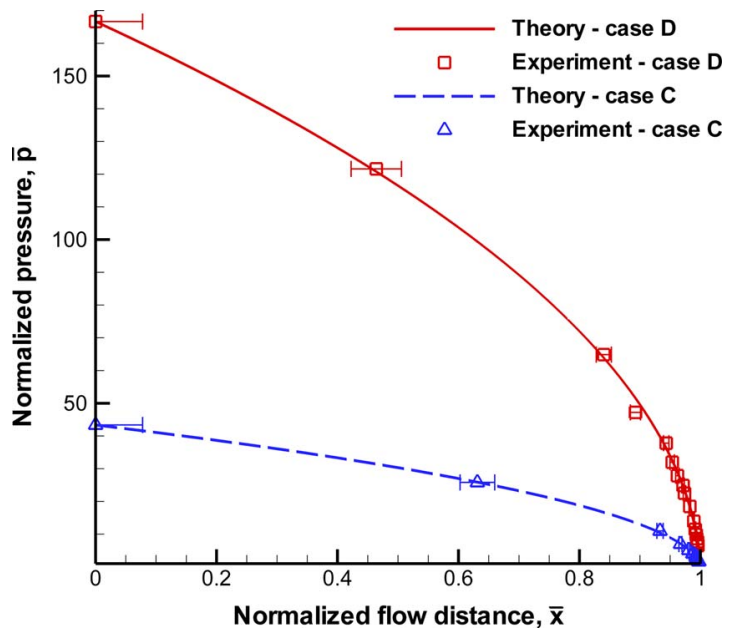

FIG. 9. (Color online) Streamwise pressure distributions deduced for two of the experimental cases.

maximum pressure ratio for that case. The uncertainty in $\bar{x}$ in Eq. (43) may be expressed as

$$
\Delta \bar{x}=\sqrt{\left[\frac{Q_{P}}{Q_{P} \max }\right]^{2}\left[\left(\frac{\Delta Q_{P}}{Q_{P}}\right)^{2}+\left(\frac{\Delta Q_{P} \max }{Q_{P} \max }\right)^{2}\right]},
$$

where $Q_{P}$ and $Q_{P}$ max represent the mass flow rate at pressure ratios of $P$ and $P_{\max }$, respectively. The two relative uncertainties in Eq. (44) for mass flow rate, i.e., $\left(\Delta Q_{P} / Q_{P}\right)$ and $\left(\Delta Q_{P \max } / Q_{P \text { max }}\right)$, are less than $5.5 \%$ according to the previous analysis.

Figure 9 shows the streamwise pressure distributions for cases $\mathrm{C}$ and $\mathrm{D}$ from the experiments (see Table I). The pressure decreases as gas flows downstream and significant pressure loss occurs near the outlet due to rapid volume expansion of the gas flow. The nonlinear distribution of pressure along the flow distance clearly shows the effect of flow compressibility. Theoretical results predicted using Eq. (25) agree quite well the experimental data.

Figure 10 shows the normalized pressure difference $\Delta \bar{p} / \bar{p}_{\text {slip }}$ calculated using the model developed here and the slip flow model, where $\Delta \bar{p}=\bar{p}-\bar{p}_{\text {slip }}$ and $\bar{p}$ and $\bar{p}_{\text {slip }}$ are calculated using Eqs. (25) and (28), respectively. It is apparent that the current model predicts a lower pressure than does the slip model (as the values in Fig. 10 are negative), especially in the region near the outlet where the rarefaction effect is the greatest. After reaching its minimum value very close to the exit, the normalized pressure difference rises quickly to zero at the exit, $\bar{x}=1$. This is because both in the current model and in the slip model the normalized pressure is fixed at the same value, i.e., 1 , at the exit. The experimental data, which are calculated as $\left(\bar{p}_{\text {expt }}-\bar{p}_{\text {slip }}\right) / \bar{p}_{\text {slip }}\left(\bar{p}_{\text {expt }}\right.$ is the experimental normalized pressure), are also presented in the figure. Although the experimental data exhibit some scatter, it is still evident that the measured pressures are lower than those from the slip flow model, as evidenced by the data being smaller than zero in Fig. 10, and agree much better with the current model. It is also noted that the streamwise pressure distribution agrees well with that predicted by the slip model in the region near the inlet $(\bar{x}=0)$, as revealed by the value of 


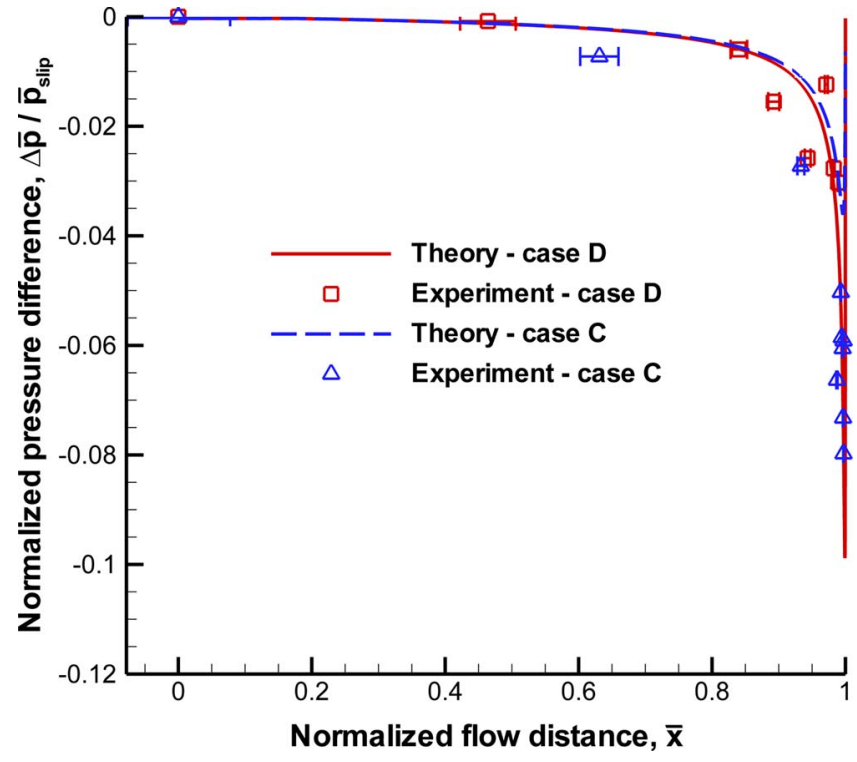

FIG. 10. (Color online) Normalized pressure difference $\Delta \bar{p} / \bar{p}_{\text {slip }}$ between the slip flow model and the current model predictions.

$\Delta \bar{p} / \bar{p}_{\text {slip }}$ being close to zero, while it deviates significantly in a region close to the outlet. This indicates that both the slip and transition flow regimes are present in the cases considered here, and that the current model applies well to flows with multiple flow regimes coexisting along the flow direction.

\section{CONCLUSIONS}

A theoretical model applicable for rarefied gas flow in long tubes with various pressure ratios between the inlet and outlet is developed. Due to significant pressure decrease along the tube, the Knudsen number changes greatly and multiple flow regimes may exist in a single tube. The new model incorporates the influence of pressure ratio and is capable of predicting gas flow covering either single or multiple flow regimes along the flow direction.

An experimental setup was designed to study rarefied gas flow subject to different pressure ratios ranging from 3 to 170. Knudsen numbers tested ranged from 0.05 to 1.08 and were mainly in the transition flow regime. The maximum uncertainty in the experimentally measured mass flow rate was $5.5 \%$.

Comparison between predictions from the current model and the experimental data showed very good agreement in terms of the mass flow rate and streamwise pressure distribution. Predictions from the current model are shown to be substantially improved in terms of agreement with experiment relative to a slip flow model.

\section{ACKNOWLEDGMENTS}

Funding for this work from the National Science Foundation is gratefully acknowledged. The support of Dr. Lei Tang is appreciated.

\section{APPENDIX: EXPRESSION FOR THE SECOND-ORDER CONSTITUTIVE TENSOR}

The second-order constitutive tensor in the Burnett equation at a constant temperature is

$$
\begin{aligned}
{[\sigma]=} & \frac{\mu^{2}}{p}\left\{\omega_{1}(\nabla \cdot U) \overline{\nabla U}+\omega_{2}\left[-\overline{\nabla\left(\frac{\nabla p}{\rho}\right)}-\overline{\nabla U \cdot \nabla U}\right.\right. \\
& \left.-2 \overline{\nabla U \cdot \overline{\nabla U}}]+\omega_{6} \overline{\nabla U \cdot \nabla U}\right\},
\end{aligned}
$$

where

$$
[\sigma]=\left[\begin{array}{lll}
\sigma_{r r} & \sigma_{\theta r} & \sigma_{x r} \\
\sigma_{r \theta} & \sigma_{\theta \theta} & \sigma_{x \theta} \\
\sigma_{r x} & \sigma_{\theta x} & \sigma_{x x}
\end{array}\right],
$$

and each component in this tensor may be written as

$$
\begin{aligned}
\sigma_{x r}= & \sigma_{r x}=\frac{\mu^{2}}{p}\left\{\frac{\omega_{1}}{2}\left(\frac{\partial u}{\partial r}+\frac{\partial v}{\partial x}\right)\left(\frac{\partial v}{\partial r}+\frac{v}{r}+\frac{\partial u}{\partial x}\right)-\omega_{2} \frac{R T}{2}\left[\frac{\partial}{\partial r}\left(\frac{1}{p} \frac{\partial p}{\partial x}\right)+\frac{\partial}{\partial x}\left(\frac{1}{p} \frac{\partial p}{\partial r}\right)\right]-\omega_{2} \frac{1}{2}\left(\frac{\partial u}{\partial x} \frac{\partial u}{\partial r}+\frac{\partial v}{\partial x} \frac{\partial v}{\partial r}+\frac{\partial u}{\partial x} \frac{\partial v}{\partial x}+\frac{\partial u}{\partial r} \frac{\partial v}{\partial r}\right)\right. \\
& \left.-\omega_{2}\left[\frac{7}{6}\left(\frac{\partial u}{\partial x} \frac{\partial u}{\partial r}+\frac{\partial v}{\partial x} \frac{\partial v}{\partial r}\right)+\frac{1}{6}\left(\frac{\partial u}{\partial x} \frac{\partial v}{\partial x}+\frac{\partial u}{\partial r} \frac{\partial v}{\partial r}\right)+\frac{1}{3} \frac{v}{r}\left(\frac{\partial u}{\partial r}+\frac{\partial v}{\partial x}\right)\right]+\omega_{6} \frac{1}{6}\left(\frac{\partial u}{\partial r}+\frac{\partial v}{\partial x}\right)\left(\frac{\partial u}{\partial x}+\frac{\partial v}{\partial r}-2 \frac{v}{r}\right)\right\}, \\
\sigma_{r r}= & \frac{\mu^{2}}{p}\left\{\omega_{1}\left[\frac{2}{3} \frac{\partial v}{\partial r}-\frac{1}{3} \frac{\partial u}{\partial x}-\frac{1}{3} \frac{v}{r}\right]\left(\frac{\partial v}{\partial r}+\frac{v}{r}+\frac{\partial u}{\partial x}\right)-\omega_{2} R T\left[\frac{2}{3} \frac{\partial}{\partial r}\left(\frac{1}{p} \frac{\partial p}{\partial r}\right)-\frac{1}{3} \frac{1}{r p} \frac{\partial p}{\partial r}-\frac{1}{3} \frac{\partial}{\partial x}\left(\frac{1}{p} \frac{\partial p}{\partial x}\right)\right]-\omega_{2}\left[\frac{2}{3}\left(\frac{\partial v}{\partial r}\right)^{2}\right.\right. \\
& \left.+\frac{1}{3} \frac{\partial u}{\partial r} \frac{\partial v}{\partial x}-\frac{1}{3}\left(\frac{v}{r}\right)^{2}-\frac{1}{3}\left(\frac{\partial u}{\partial x}\right)^{2}\right]-2 \omega_{2}\left\{\frac{1}{2}\left(\frac{\partial u}{\partial r}\right)^{2}+\frac{2}{3}\left(\frac{\partial v}{\partial r}\right)^{2}-\frac{1}{3} \frac{\partial u}{\partial x} \frac{\partial v}{\partial r}+\frac{1}{2} \frac{\partial u}{\partial r} \frac{\partial v}{\partial x}-\frac{1}{3} \frac{\partial v}{\partial r} \frac{v}{r}-\left[\frac{2}{9}\left(\frac{\partial u}{\partial x}\right)^{2}+\frac{1}{6}\left(\frac{\partial u}{\partial r}\right)^{2}\right.\right. \\
& \left.\left.+\frac{2}{9}\left(\frac{\partial v}{\partial r}\right)^{2}+\frac{1}{6}\left(\frac{\partial v}{\partial x}\right)^{2}-\frac{2}{9} \frac{\partial u}{\partial x} \frac{\partial v}{\partial r}+\frac{1}{3} \frac{\partial u}{\partial r} \frac{\partial v}{\partial x}-\frac{2}{9} \frac{v}{r}\left(\frac{\partial u}{\partial x}+\frac{\partial v}{\partial r}\right)+\frac{2}{9}\left(\frac{v}{r}\right)^{2}\right]\right\}+\omega_{6}\left\{\left(\frac{2}{3} \frac{\partial v}{\partial r}-\frac{1}{3} \frac{\partial u}{\partial x}-\frac{1}{3} \frac{v}{r}\right)^{2}+\frac{1}{4}\left(\frac{\partial u}{\partial r}+\frac{\partial v}{\partial x}\right)^{2}\right. \\
& \left.\left.-\frac{2}{9}\left[\left(\frac{\partial u}{\partial x}\right)^{2}+\left(\frac{\partial v}{\partial r}\right)^{2}-\frac{\partial u}{\partial x} \frac{v}{r}-\frac{\partial v}{\partial r} \frac{v}{r}-\frac{\partial u}{\partial x} \frac{\partial v}{\partial r}+\left(\frac{v}{r}\right)^{2}\right]-\frac{1}{6}\left(\frac{\partial u}{\partial r}+\frac{\partial v}{\partial x}\right)^{2}\right\}\right\},
\end{aligned}
$$




$$
\begin{aligned}
\sigma_{\theta \theta}= & \frac{\mu^{2}}{p}\left\{\omega_{1}\left[\frac{2}{3} \frac{v}{r}-\frac{1}{3} \frac{\partial u}{\partial x}-\frac{1}{3} \frac{\partial v}{\partial r}\right]\left(\frac{\partial v}{\partial r}+\frac{v}{r}+\frac{\partial u}{\partial x}\right)-\omega_{2} R T\left[\frac{2}{3} \frac{1}{r p} \frac{\partial p}{\partial r}-\frac{1}{3} \frac{\partial}{\partial r}\left(\frac{1}{p} \frac{\partial p}{\partial r}\right)-\frac{1}{3} \frac{\partial}{\partial x}\left(\frac{1}{p} \frac{\partial p}{\partial x}\right)\right]-\omega_{2}\left[\frac{2}{3}\left(\frac{v}{r}\right)^{2}-\frac{2}{3} \frac{\partial u}{\partial r} \frac{\partial v}{\partial x}\right.\right. \\
& \left.-\frac{1}{3}\left(\frac{\partial v}{\partial r}\right)^{2}-\frac{1}{3}\left(\frac{\partial u}{\partial x}\right)^{2}\right]-2 \omega_{2}\left(\frac{2}{3}\left(\frac{v}{r}\right)^{2}-\frac{1}{3} \frac{\partial u}{\partial x} \frac{v}{r}-\frac{1}{3} \frac{\partial v}{\partial r} \frac{v}{r}-\left[\frac{2}{9}\left(\frac{\partial u}{\partial x}\right)^{2}+\frac{1}{6}\left(\frac{\partial u}{\partial r}\right)^{2}+\frac{2}{9}\left(\frac{\partial v}{\partial r}\right)^{2}+\frac{1}{6}\left(\frac{\partial v}{\partial x}\right)^{2}-\frac{2}{9} \frac{\partial u}{\partial x} \frac{\partial v}{\partial r}\right.\right. \\
& \left.\left.+\frac{1}{3} \frac{\partial u}{\partial r} \frac{\partial v}{\partial x}-\frac{2}{9} \frac{v}{r}\left(\frac{\partial u}{\partial x}+\frac{\partial v}{\partial r}\right)+\frac{2}{9}\left(\frac{v}{r}\right)^{2}\right]\right\}+\omega_{6}\left\{\left(\frac{2}{3} \frac{v}{r}-\frac{1}{3} \frac{\partial u}{\partial x}-\frac{1}{3} \frac{\partial v}{\partial r}\right)^{2}-\frac{2}{9}\left[\left(\frac{\partial u}{\partial x}\right)^{2}+\left(\frac{\partial v}{\partial r}\right)^{2}-\frac{\partial u}{\partial x} \frac{v}{r}-\frac{\partial v}{\partial r} \frac{v}{r}-\frac{\partial u}{\partial x} \frac{\partial v}{\partial r}\right.\right. \\
& \left.\left.\left.+\left(\frac{v}{r}\right)^{2}\right]-\frac{1}{6}\left(\frac{\partial u}{\partial r}+\frac{\partial v}{\partial x}\right)^{2}\right\}\right\}, \\
\sigma_{x x}= & \frac{\mu^{2}}{p}\left\{\omega_{1}\left[\frac{2}{3} \frac{\partial u}{\partial x}-\frac{1}{3}\left(\frac{\partial v}{\partial r}+\frac{v}{r}\right)\right]\left(\frac{\partial v}{\partial r}+\frac{v}{r}+\frac{\partial u}{\partial x}\right)-\omega_{2} R T\left[\frac{2}{3} \frac{\partial}{\partial x}\left(\frac{1}{p} \frac{\partial p}{\partial x}\right)^{-\frac{1}{3 r p}} \frac{\partial p}{\partial r}-\frac{1}{3} \frac{\partial}{\partial r}\left(\frac{1}{p} \frac{\partial p}{\partial r}\right)\right]-\omega_{2}\left[\frac{2}{3}\left(\frac{\partial u}{\partial x}\right)^{2}\right.\right. \\
& \left.+\frac{1}{3} \frac{\partial u}{\partial r} \frac{\partial v}{\partial x}-\frac{1}{3}\left(\frac{v}{r}\right)^{2}-\frac{1}{3}\left(\frac{\partial v}{\partial r}\right)^{2}\right]-2 \omega_{2}\left\{\frac{1}{2}\left(\frac{\partial v}{\partial x}\right)^{2}+\frac{2}{3}\left(\frac{\partial u}{\partial x}\right)^{2}-\frac{1}{3} \frac{\partial u}{\partial x} \frac{\partial v}{\partial r}+\frac{1}{2} \frac{\partial u}{\partial r} \frac{\partial v}{\partial x}-\frac{1}{3} \frac{\partial u}{\partial x} \frac{v}{r}-\left[\frac{2}{9}\left(\frac{\partial u}{\partial x}\right)^{2}+\frac{1}{6}\left(\frac{\partial u}{\partial r}\right)^{2}\right.\right. \\
& \left.\left.+\frac{2}{9}\left(\frac{\partial v}{\partial r}\right)^{2}+\frac{1}{6}\left(\frac{\partial v}{\partial x}\right)^{2}-\frac{2}{9} \frac{\partial u}{\partial x} \frac{\partial v}{\partial r}+\frac{1}{3} \frac{\partial u}{\partial r} \frac{\partial v}{\partial x}-\frac{2}{9} \frac{v}{r}\left(\frac{\partial u}{\partial x}+\frac{\partial v}{\partial r}\right)^{2}+\frac{2}{9}\left(\frac{v}{r}\right)^{2}\right]\right\}+\omega_{6}\left\{\left(\frac{2}{3} \frac{\partial u}{\partial x}-\frac{1}{3} \frac{\partial v}{\partial r}-\frac{1}{3} \frac{v}{r}\right)^{2}+\frac{1}{4}\left(\frac{\partial u}{\partial r}\right.\right. \\
& \left.\left.\left.+\frac{\partial v}{\partial x}\right)^{2}-\frac{2}{9}\left[\left(\frac{\partial u}{\partial x}\right)^{2}+\left(\frac{\partial v}{\partial r}\right)^{2}-\frac{\partial u}{\partial x} \frac{v}{r}-\frac{\partial v}{\partial r} \frac{v}{r}-\frac{\partial u}{\partial x} \frac{\partial v}{\partial r}+\left(\frac{v}{r}\right)^{2}\right]-\frac{1}{6}\left(\frac{\partial u}{\partial r}+\frac{\partial v}{\partial x}\right)^{2}\right\}\right\}, \\
&
\end{aligned}
$$

$\sigma_{r \theta}=\sigma_{\theta r}=0$

$\sigma_{\theta x}=\sigma_{x \theta}=0$

${ }^{1}$ S. A. Schaaf and P. L. Chambre, Flow of Rarefied Gases (Princeton University Press, Princeton, 1961).

${ }^{2}$ R. Edwards, "Low density flows through tubes and nozzles," in Rarefied Gas Dynamics, edited by J. Potter (AIAA, New York, 1977), Part I, p. 199. ${ }^{3}$ E. Arkilic, M. Schmidt, and K. Breuer, "Gaseous flow in microchannels," in Application of Microfabrication to Fluid Mechanics (ASME, New York, 2004), ASME Winter Annual Meeting, Chicago, IL, 1994, p. 57.

${ }^{4}$ J. Harley, Y. Huang, H. Bau, and J. N. Zemel, "Gas flow in microchannels," J. Fluid Mech. 284, 257 (1995).

A. Beskok and G. Karniadakis, "Simulation of heat and momentum transfer in complex microgeometries," J. Thermophys. Heat Transfer 8, 647 (1994).

${ }^{6}$ K. Pong, C. Ho, J. Liu, and Y. Tai, "Non-linear pressure distribution in uniform microchannels," in Application of Microfabrication to Fluid Mechanics (ASME, New York, 1994), ASME Winter Annual Meeting, Chicago, IL, 1994, p. 51.

${ }^{7}$ R. Prud'homme, T. Chapman, and J. Bowen, "Laminar compressible flow in a tube," Appl. Sci. Res. 43, 67 (1986).

${ }^{8}$ E. Piekos and K. Breuer, "Numerical modeling of micromechanical devices using the direct simulation Monte Carlo method," J. Fluids Eng. 118, 4641996.

${ }^{9}$ G. Bird, Molecular Gas Dynamics and the Direct Simulation of Gas Flows (Clarendon, Oxford, 1994).

${ }^{10}$ R. G. Lord, "Direct simulation Monte Carlo calculations of rarefied flows with incomplete surface accommodation," J. Fluid Mech. 239, 449 (1992).

${ }^{11}$ M. A. Gallis, J. R. Torczynski, and D. J. Rader, "An approach for simulating the transport of spherical particles in a rarefied gas flow via the direct simulation Monte Carlo method," Phys. Fluids 13, 3482 (2001).

${ }^{12} \mathrm{~K}$. Yamamoto, H. Takeuchi, and T. Hyakutake, "Characteristics of reflected gas molecules at a solid surface," Phys. Fluids 18, 046103 (2006).

${ }^{13}$ S. Y. Chou and D. Baganoff, "Kinetic flux-vector splitting for the NavierStokes equations," J. Comput. Phys. 130, 217 (1997).

${ }^{14}$ A. J. Lofthouse, I. D. Boyd, and M. J. Wright, "Effects of continuum breakdown on hypersonic aerothermodynamics," Phys. Fluids 19, 027105 (2007).

${ }^{15} \mathrm{C}$. Cercignani, Theory and Application of the Boltzmann Equation (Elsevier-North-Holland, New York, 1975).

${ }^{16}$ M. M. Williams, Mathematical Methods in Particle Transport Theory (Butterworths, London, 1971).

${ }^{17}$ P. L. Bhatnagar, E. P. Gross, and K. Krook, "A model for collision processes in gases," Phys. Rev. 94, 511 (1954).

${ }^{18}$ T. Ohwada, Y. Sone, and K. Aoki, "Numerical analysis of the Poiseuille and thermal transpiration flows between two parallel plates on the basis of the Boltzmann equation for hard sphere molecules," Phys. Fluids A 1, 2042 (1989).

${ }^{19}$ A. B. Huang, D. P. Giddens, and C. W. Bagnal, "Rarefied gas flow between parallel plates based on the discrete ordinate method," Phys. Fluids 10, 498 (1967).

${ }^{20}$ S. K. Loyalka and S. A. Hamoodi, "Poiseuille flow of a rarefied gas in a cylindrical tube: Solution of linearized Boltzmann equation," Phys. Fluids A 2, 2061 (1990).

${ }^{21}$ K. Aoki, in Rarefied Gas Dynamics: Theoretical and Computational Techniques, edited by E. P. Muntz, D. P. Weaver, and D. H. Campbell (AIAA, Washington, 1989), p. 297.

${ }^{22}$ Y. Zohar, S. Y. K. Lee, W. Y. Lee, L. Jiang, and P. Tong, "Subsonic gas flow in a straight and uniform microchannel," J. Fluid Mech. 472, 125 (2002).

${ }^{23}$ X. Zhong and G. H. Furumoto, "Augmented Burnett-equation solutions over axisymmetric blunt bodies in hypersonic flow," J. Spacecr. Rockets 32, 588 (1995).

${ }^{24} \mathrm{~S}$. Chapman and T. G. Cowling, The Mathematical Theory of NonUniform Gases, 3rd ed. (Cambridge University Press, Cambridge, 1970).

${ }^{25}$ A. Beskok and G. E. Karniadakis, "A model for flows in channels, pipes, and ducts at micro and nano scales," Microscale Thermophys. Eng. 3, 43 (1999).

${ }^{26}$ A. Beskok, G. E. Karniadakis, and W. Trimmer, "Rarefaction and compressibility effects in gas microflows," J. Fluids Eng. 118, 448 (1996).

${ }^{27}$ J. A. Goff and S. Gratch, "Low-pressure properties of water from 160 to 212 F," in Transactions of the American Society of Heating and Ventilating Engineers (ASHVE, New York, 1946), 52nd Annual Meeting of the American Society of Heating and Ventilating Engineers, New York, NY, 1946, p. 95. 